\title{
Yeast zymosan, a stimulus for TLR2 and dectin-1, induces regulatory antigen- presenting cells and immunological tolerance
}

\begin{abstract}
Stephanie Dillon, ${ }^{1}$ Sudhanshu Agrawal, ${ }^{1}$ Kaustuv Banerjee, ${ }^{1}$ John Letterio, ${ }^{2}$ Timothy L. Denning, ${ }^{1}$ Kyra Oswald-Richter, ${ }^{3}$ Deborah J. Kasprowicz, ${ }^{4}$ Kathryn Kellar, ${ }^{5}$ Jeff Pare, ${ }^{6}$ Thomas van Dyke, ${ }^{7}$ Steven Ziegler, ${ }^{4}$ Derya Unutmaz, ${ }^{3}$ and Bali Pulendran'

${ }^{1}$ Emory Vaccine Research Center and Department of Pathology, Emory University, Atlanta, Georgia, USA. ${ }^{2}$ Immunoregulation Section, Lab of Cell Regulation and Carcinogenesis, Center for Cancer Research, National Cancer Institute, NIH, Bethesda, Maryland, USA. ${ }^{3}$ Department of Microbiology and Immunology, Vanderbilt University Medical School, Nashville, Tennessee, USA. ${ }^{4}$ Immunology Program, Benaroya Research Institute, Seattle, Washington, USA. ${ }^{5}$ Scientific Resources Program, National Center for Infectious Diseases, Centers for Disease Control and Prevention, Atlanta, Georgia, USA. ${ }^{6}$ Yerkes National Primate Research Center, Emory University, Atlanta, Georgia, USA. Department of Periodontology and Oral Biology, Boston University School of Medicine, Boston, Massachusetts, USA.
\end{abstract}

\begin{abstract}
Emerging evidence suggests critical roles for APCs in suppressing immune responses. Here, we show that zymosan, a stimulus for TLR2 and dectin-1, regulates cytokine secretion in DCs and macrophages to induce immunological tolerance. First, zymosan induces DCs to secrete abundant IL-10 but little IL-6 and IL-12(p70). Induction of IL-10 is dependent on TLR2- and dectin-1-mediated activation of ERK MAPK via a mechanism independent of the activation protein 1 (AP-1) transcription factor c-Fos. Such DCs stimulate antigen-specific CD4 ${ }^{+} \mathrm{T}$ cells poorly due to IL-10 and the lack of IL-6. Second, zymosan induces $\mathrm{F} 4-\mathbf{8 0}^{+}$macrophages in the splenic red pulp to secrete TGF- $\beta$. Consistent with these effects on APCs, injection of zymosan plus OVA into mice results in OVAspecific $T$ cells that secrete little or no Th1 or Th2 cytokines, but secrete robust levels of IL-10, and are unresponsive to challenge with OVA plus adjuvant. Finally, coinjection of zymosan with OVA plus LPS suppresses the response to OVA via a mechanism dependent on IL-10, TGF- $\beta$, and lack of IL-6. Together, our data demonstrate that zymosan stimulates IL-10 ${ }^{+} \mathrm{IL}-12(\mathrm{p} 70)-\mathrm{IL}-6^{\text {low }}$ regulatory DCs and TGF- $\beta^{+}$macrophages to induce immunological tolerance. These data suggest several targets for pharmacological modulation of immune responses in various clinical settings.
\end{abstract}

\section{Introduction}

Sensing a pathogen is the first, critical step in launching an immune defense. The immune system has evolved an elaborate system of pathogen surveillance, the so-called pathogen-recognition receptors (PRRs), which can recognize highly conserved molecular signatures contained in microbes and then decode this information to elicit an immune response (1-3). The prototypic examples of PRRs are the TLRs $(4,5)$ and the C-type lectins $(6-8)$, which are expressed on and in DCs. Although DCs were initially thought to be important in initiating immune responses, more recent evidence points to a central role for them in tuning the quality of the immune response as well as in suppressing immune responses $(1,2)$. For example, there is now much evidence that triggering different TLRs on DCs results in distinct programs of gene expression and cytokine secretion that differentially regulate the type of adaptive immune responses (2, 4, 8-14). Furthermore, emerging evidence suggests that signaling through DC-SIGN, a C-type lectin, results in impaired DC maturation and antiinflammatory responses (15). Thus, determining the immunological consequences of triggering distinct PRRs on DCs might offer novel strategies for therapeutic immune intervention in various clinical settings. With this perspective, we embarked on a systematic

Nonstandard abbreviations used: AP-1, activation protein-1; B6.PL, B6.PL.Thy-1ª; IFA, incomplete Freund's adjuvant; phospho, phosphorylated.

Conflict of interest: The authors have declared that no conflict of interest exists. Citation for this article: J. Clin. Invest. 116:916-928 (2006). doi:10.1172/JCI27203. screening of several PRR ligands for their effects on DCs and the adaptive immune response. In the present report, we describe the unique effects of zymosan, a yeast cell wall derivative which is recognized by dectin-1, a C-type lectin receptor for $\beta$-glucans (16-18) expressed in murine (18) and human (19) DCs in conjunction with TLR2 (20). Our data suggest that zymosan induces regulatory DCs, which secrete abundant IL-10 but little or no IL-6 and IL-12(p70) and induce impaired $\mathrm{T}$ cell responses. Such regulatory DCs appear to be induced via activation of TLR2- and dectin-1-dependent activation of ERK MAPK, which promotes IL-10 production. Furthermore, zymosan induces splenic $\mathrm{F} 4-80^{+}$macrophages to secrete TGF- $\beta$, another immunosuppressive cytokine. Consistent with this, injection of antigen plus zymosan into mice results in a transient but weak activation of antigen-specific $T$ cells, which are resistant to further stimulation. Further, coinjection of zymosan with LPS plus antigen results in reduced antigen-specific $T$ cell proliferation compared with LPS plus antigen injection alone. Thus zymosan appears to program DCs and macrophages to suppress immune responses via multiple mechanisms.

\section{Results}

Zymosan induces DCs to secrete robust IL-10 but little or no IL-12(p70) and $I L-6$. We first determined the responses of human and murine DCs to zymosan. Human monocyte-derived DCs cultured for 24 hours with either zymosan or LPS (a TLR4 ligand) were observed to upregulate costimulatory molecules CD80 and CD86 as well as the maturation marker CD83 (Figure 1A). There are presently con- 


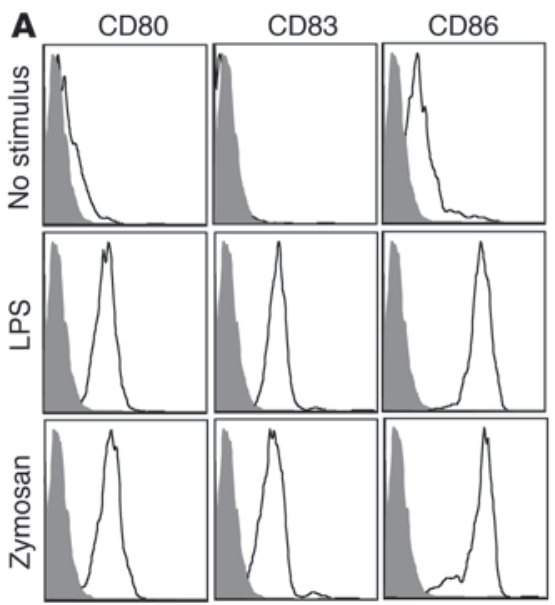

D

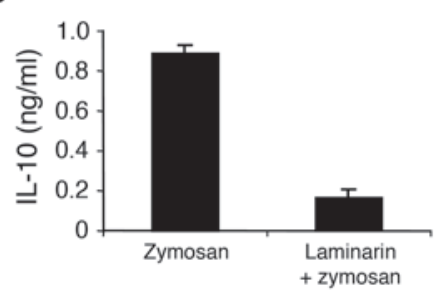

B

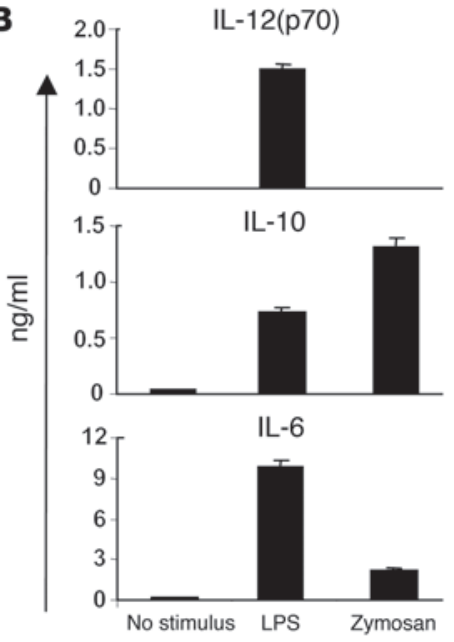

E

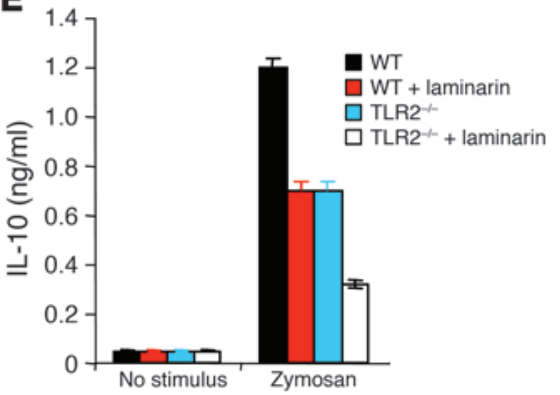

c
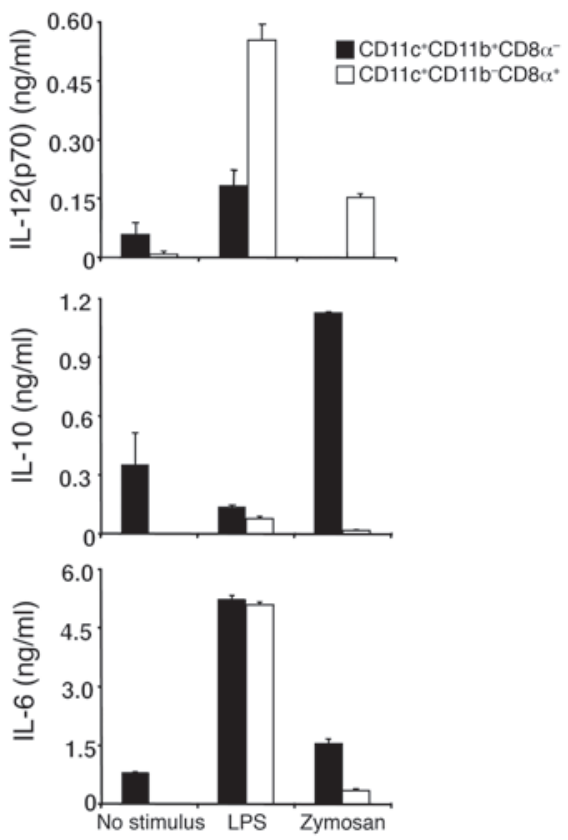

Figure 1

Zymosan induces IL-10 in human and murine DCs via a mechanism involving dectin-1. (A) Immature, monocyte-derived DCs were cultured for 24-48 hours with E. coli LPS $(1 \mu \mathrm{g} / \mathrm{ml})$ or zymosan $(50 \mu \mathrm{g} / \mathrm{ml})$, and flow cytometric analyses of the expression of the costimulatory molecules CD80 and CD86 and the maturation marker CD83 were performed. Filled histograms show isotype; open histograms represent marker. Data are representative of 10 experiments. (B) Secretion of cytokines in the culture supernatants was measured by ELISA. Data are representative of 18 experiments. (C) Murine, splenic CD11 $+C D 11 b+C D 8 \alpha^{-}$, and CD11c $+C D 11 b-C D 8 \alpha^{+}$DC subsets were isolated by flow cytometry and cultured in vitro with E. coli LPS or zymosan for 24-48 hours in the presence of a CD40-L-expressing cell line. Cytokines were analyzed by ELISA. Data are representative of 10 experiments. (D) Immature, monocyte-derived DCs were cultured for $24-48$ hours with laminarin (200 $\mu \mathrm{g} / \mathrm{ml})$ plus zymosan or with zymosan alone. IL-10 levels were measured after 24 hours by ELISA. Data are representative of 4 experiments. (E) CD11C ${ }^{+}$ murine splenic DCs were cultured with laminarin $(200 \mu \mathrm{g} / \mathrm{ml})$ plus zymosan and CD40-L-expressing fibroblasts. IL-10 levels were measured after 24 hours by ELISA. Data are representative of 7 experiments.

flicting reports regarding the cytokine profiles induced by zymosan. Although previous work suggests that zymosan stimulates DCs to produce proinflammatory cytokines, including IL-12(p40) (20), more recent studies have shown that zymosan also stimulated DCs to produce robust IL-10 $(21,22)$. Therefore, it was important to determine the induction of these cytokines in our system. In human monocyte-derived DCs, while LPS induced abundant levels of IL-12(p70), IL-6, and IL-10, zymosan induced barely detectable levels of IL-12(p70) and lower levels of IL-6 but abundant levels of IL-10 (Figure 1B). This was consistent with data from the murine system, in which splenic CD $11 \mathrm{c}^{+} \mathrm{CD} 11 \mathrm{~b}^{+} \mathrm{CD} 8 \alpha^{-}$and $\mathrm{CD} 11 \mathrm{c}^{+} \mathrm{CD} 11 \mathrm{~b}^{-} \mathrm{CD} 8 \alpha^{+} \mathrm{DCs}(2,3)$ were isolated by flow cytometry from Flt3 ligand-treated mice (3) and cultured with LPS or zymosan in the presence of a CD40 ligand-expressing fibroblast cell line $(14,21)$, which is known to amplify cytokine production in this system (21). LPS induced much higher levels of IL-12(p70) and IL-6 than zymosan from both DC subsets (Figure 1C). As observed previously (14), LPS preferentially induced IL-12(p70) from the CD $11 c^{+} C D 11 b^{-C D} 8 \alpha^{+}$DCs. However, consistent with the data from human DCs, zymosan induced profoundly higher levels of
IL-10 than LPS, preferentially in CD $11 c^{+} C D 11 b^{+} C D 8 \alpha^{-}$DCs (Figure $1 C)$. Additionally, similar profiles were seen in the absence of CD40 ligand cells albeit with undetectable levels of IL-12(p70) (Supplemental Figure 1; supplemental material available online with this article; doi:10.1172/JCI27203DS1). Furthermore, kinetic analysis of cytokine production suggested that, while zymosan was more rapid at inducing IL-10 relative to LPS, the latter was more rapid at inducing IL-6 and IL-12(p70) (Supplemental Figure 2). Thus, LPS and zymosan induce very distinct cytokine responses in human and murine DCs. It is noteworthy that IL-6, a cytokine that was recently shown to suppress the induction of regulatory $\mathrm{T}$ cells (23), was induced poorly by zymosan.

We then investigated the receptors through which these 2 microbial stimuli mediate their distinct effects. As observed previously, all the effects of LPS were mediated through TLR4 (data not shown). In the case of zymosan, 2 recognition receptors have been described. Dectin-1, a C-type lectin (16-19), is expressed on murine splenic DCs (18) and on human DCs (19) as well as on macrophages and neutrophils and mediates attachment and ingestion of zymosan. Dectin-1 recognition of zymosan appears 

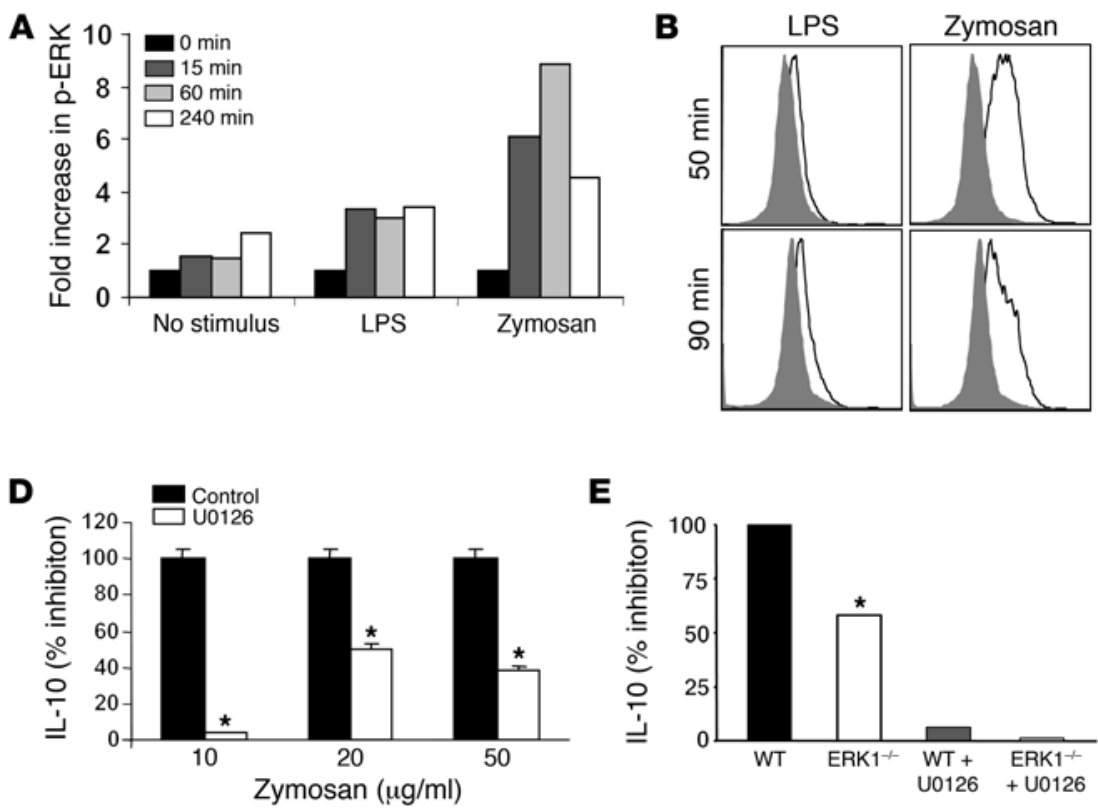

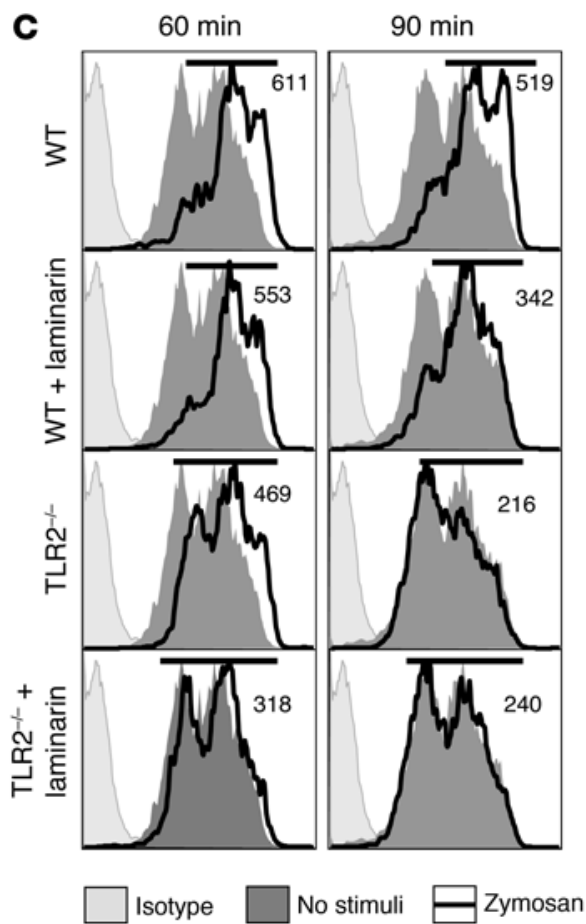

Figure 2

Zymosan induces IL-10 in DCs via a mechanism dependent on ERK MAPK. (A) On day 6, human monocyte-derived DCs were cultured with LPS or zymosan for $0,15,60$, or 240 minutes. At each time point, the expression of phosphorylated and total ERK was evaluated by ELISA. Data are presented as the fold increases in the phosphorylated to total protein ratios relative to the 0 minute value. Data are representative of 4 experiments. (B) Flow cytometric analyses of phospho-ERK expression in DCs. Filled histograms represent the staining in unstimulated DCs, and the open histograms represent staining after stimulation. Data are representative of 3 experiments. (C) Effect of blocking dectin-1- $\beta$-glucan interactions, with laminarin on ERK activation. Data are representative of 3 experiments. Numbers in histograms indicate mean fluorescent intensities of the zymosan-treated DCs. No stimulus indicates DCs cultured in medium alone for 60 or 90 minutes. (D) Effect of blocking ERK1 and 2 with U0126, a synthetic MEK1 and 2 inhibitor, on IL-10 induction by zymosan in human monocyte-derived DCs. Data are representative of 4 experiments. (E) Role of ERK1 and 2 on IL-10 induction by zymosan on CD11C ${ }^{+}$murine splenic DCs. DCs from wild-type or ERK1-l- mice were cultured in vitro in the presence of zymosan for 24-48 hours and IL-10 levels assayed by ELISA. Data are representative of 3 experiments. ${ }^{*} P<0.05$, control vs. U0126 and WT vs. ERK1-1-

to be dependent on $\beta$-glucans, which are a major component of the yeast cell wall, because laminarin (a soluble $\beta$-glucan from the brown seaweed Laminaria digitata) can block this recognition (17, 18). Consistent with this, zymosan-induced IL-10 production by human monocyte-derived DCs was significantly blocked by laminarin (Figure 1D). In mice, there was a significant diminution of IL-10 production when laminarin was used to block zymosan-dectin-1 interactions in DCs from wild-type mice (Figure 1E). In addition to dectin-1, TLR2 is also known to recognize zymosan (20, 24). DCs from TLR2-deficient mice produced lower levels of IL-10 in response to zymosan, and a further decrease in IL-10 production was observed when laminarin was used to block signaling via dectin-1 on TLR2-deficient DCs (Figure 1E). Taken together, these data suggest an important role for both dectin-1 and TLR2 in the induction of IL-10 in DCs.

Zymosan induction of IL-10 in DCs is dependent on TLR2- and dectin-1mediated activation of ERK MAPK. We next sought to gain some insights into the intracellular cell-signaling networks within DCs that might mediate zymosan-induced IL-10 production. Previous work suggested that ERK MAPK is a potent mediator of TLR2-mediated IL-10 production by DCs $(13,14)$. Human and murine DCs cultured with zymosan expressed enhanced and sustained levels of ERK1 and 2 phosphorylation relative to DCs cultured with LPS (Figure 2, A and B). Our previous work suggests that Pam-3-cys, a synthetic TLR2 ligand, induces enhanced and sustained levels of ERK phosphorylation relative to TLR4 or TLR5 ligands $(13,14)$. The involvement of TLR2 in the induction of ERK phosphorylation in response to zymosan was demonstrated by lower levels being observed in TLR2deficient DCs (Figure 2C). However, the induction of ERK phosphorylation by zymosan also appeared to be, at least in part, mediated by dectin-1, since laminarin could partially block this in wild-type DCs (Figure 2C). Crucial roles for the involvement of both dectin-1 and TLR2 in ERK phosphorylation were demonstrated by blocking dectin-1 receptors in TLR2-deficient DCs (Figure 2C).

We determined the role of ERK1 and 2 in IL-10 induction, using synthetic inhibitors of ERK activation as well as ERK1-deficient mice. In the presence of the synthetic inhibitor U0126, which inhibits MEK1 and 2, the upstream activators of ERK1 and 2, there was a profound impairment of IL-10 secretion by human DCs in response to zymosan (Figure 2D). Consistent with this, DCs from ERK1-deficient mice were significantly impaired in their ability to produce IL-10, and in the presence of U0126, there was a more profound reduction (Figure 2E). Therefore, zymosan appears to mediate IL-10 production in human and murine DCs via a mechanism involving ERK MAPK activation, and this is mediated through both TLR2 and dectin-1. 

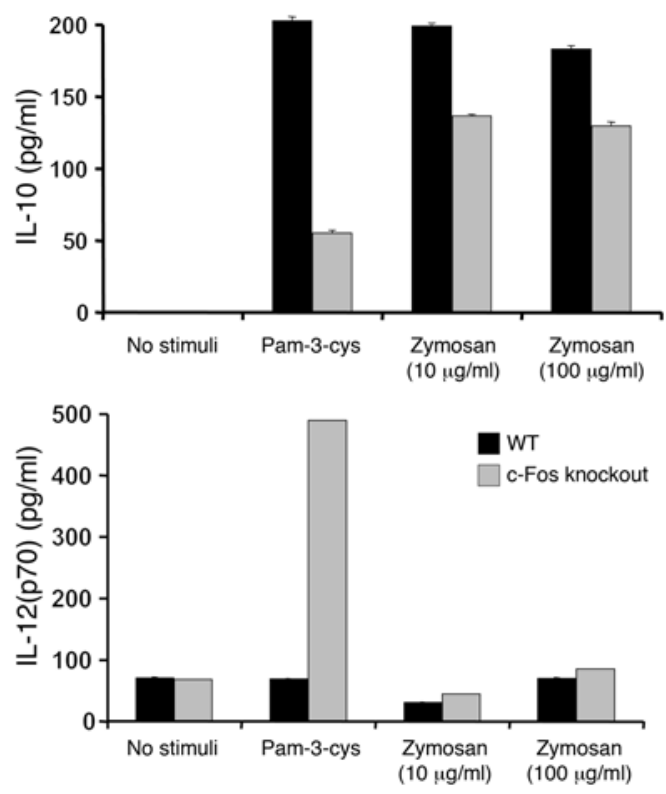

Figure 3

Zymosan induction of IL-10 in DCs is not dependent on c-Fos. CD11c ${ }^{+}$ murine splenic DCs from wild-type or c-Fos-deficient mice were stimulated for 24 hours with zymosan or Pam-3-cys $(100 \mu \mathrm{g} / \mathrm{ml})$ and CD40 ligand cells. IL-10 levels were assayed by ELISA. Data are representative of 3 experiments.

Zymosan induction of IL-10 in DCs is not dependent on c-Fos. We next addressed the question of what downstream transcription factors might mediate the induction of IL-10 in DCs by zymosan. Our previous work suggests that the synthetic TLR2 ligand Pam-3-cys, which induces enhanced and sustained ERK activation, results in phosphorylation and stabilization of the activation protein 1 (AP-1) transcription factor c-Fos, which plays a critical role in regulating IL-12(p70) and IL-10 (13, 14). Therefore, we determined whether c-Fos also plays a role in regulating IL-10 induction by zymosan (Figure 3). Thus, DCs from wild-type or c-Fos mice were cultured with zymosan or Pam-3-cys in the presence of a CD40 ligand-expressing fibroblast cell line. As reported previously (14), c-Fos-deficient DCs produced much lower levels of IL-10 and greater levels of IL-12(p70) in response to Pam3-cys (Figure 3). Interestingly, however, c-Fos did not appear to play a role in regulating these cytokines in response to zymosan, suggesting the involvement of additional transcriptional factors (Figure 3).

$I L-10$ regulates the induction of $T N F-\alpha, I L-12$, and IL-6 in DCs. IL-10 is known to be an antiinflammatory cytokine that inhibits the produc-

\section{Figure 4}

IL- 10 regulates the induction of TNF- $\alpha$, IL-12, and IL-6 in DCs. Wild-type or IL-10-deficient murine splenic DCs were cultured with zymosan $(100 \mu \mathrm{g} /$ $\mathrm{ml}$ ) and CD40 ligand cells for 24 hours, and levels of cytokines were assayed by ELISA.
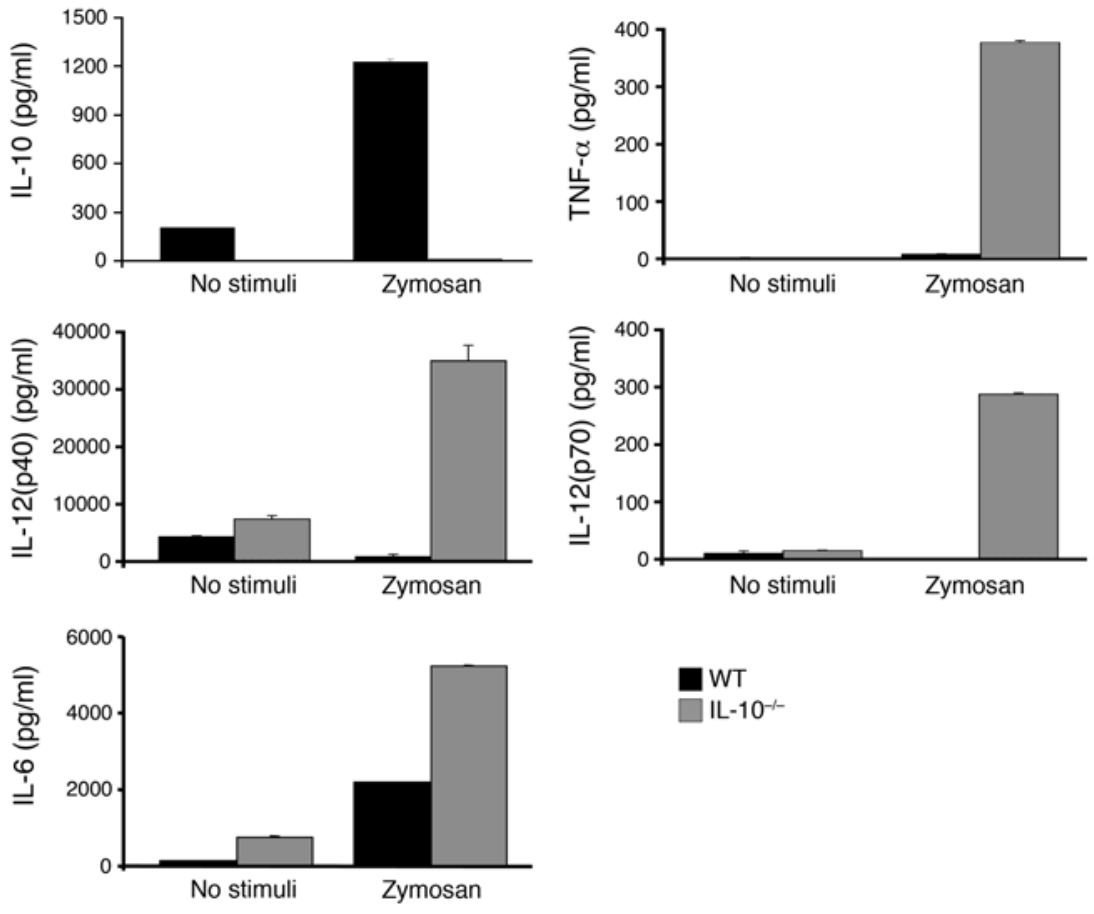

WT

IL $-10^{-/-}$ 

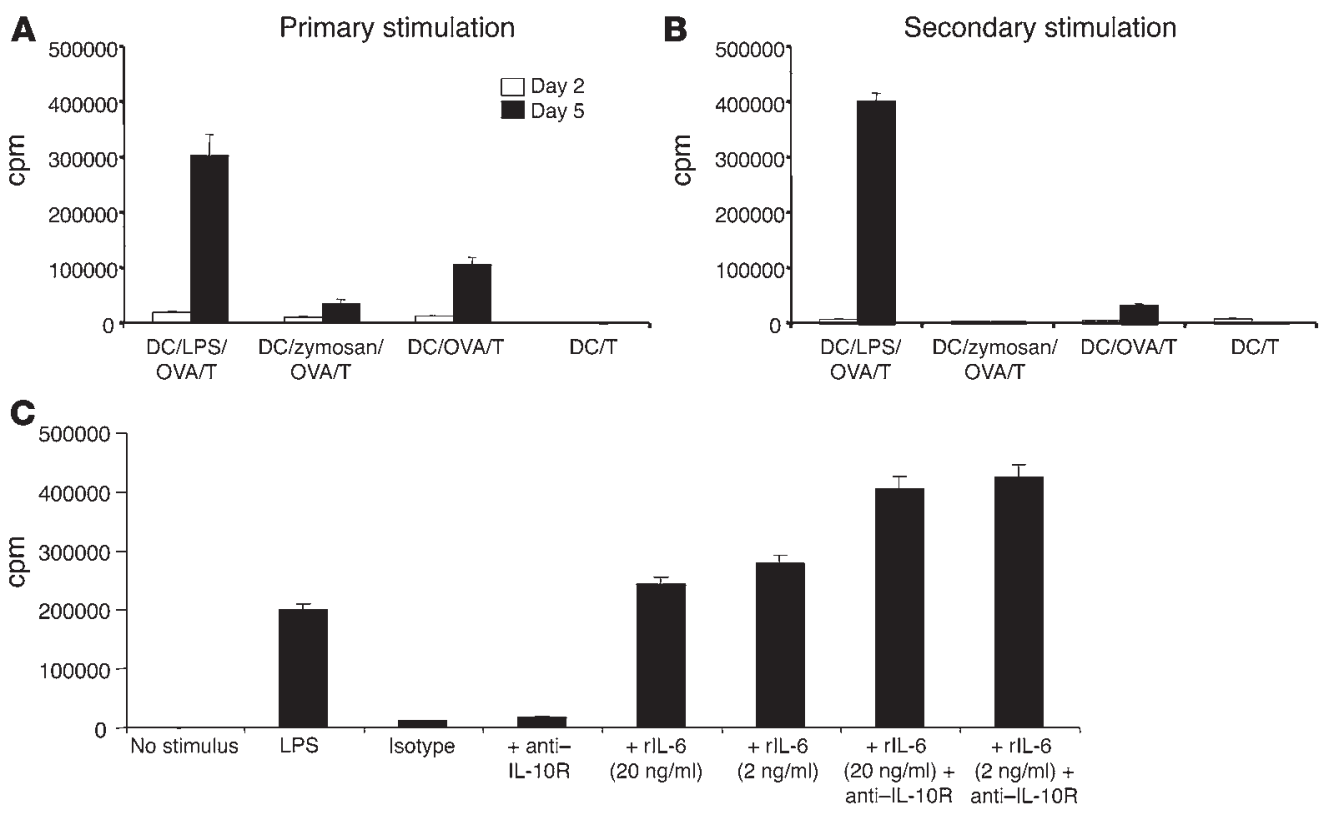

+ Zymosan

Figure 5

Zymosan-treated DCs induce impaired activation of antigen-specific T cells in vitro via a mechanism dependent on IL-10 and lack of IL-6. (A) CD11 $\mathrm{C}^{+}$murine splenic DCs (10,000/well) were cultured with naive CD4+CD62L ${ }^{+}$OT-II T cells (50,000/well), OVA $323-339$ peptide (10 $\left.\mu \mathrm{g} / \mathrm{ml}^{+}\right)$, and either LPS $(1 \mu \mathrm{g} / \mathrm{ml})$ or zymosan $(10 \mu \mathrm{g} / \mathrm{ml})$ for 2 or 5 days (primary stimulation). (B) T cells were restimulated with plate-bound anti-CD3 and soluble anti-CD28 for an additional 3 days and levels of proliferation determined by incorporation of tritiated thymidine (cpm). (C) Recombinant murine IL-6 (rIL-6) or neutralizing anti-IL-10 receptor (anti-IL-10R) antibodies were added into cultures to determine the role of these cytokines in $\mathrm{T}$ cell proliferation induced by zymosan.

in the primary response (Figure 5A), T cells stimulated with zymosan-treated DCs proliferated even less than those stimulated with DCs pulsed with OVA alone (Figure 5B).

We then determined whether the impaired responsiveness of $\mathrm{T}$ cells stimulated with zymosan-pulsed DCs was caused by robust induction of IL-10 or by the lack of high levels of proinflammatory cytokines from such DCs (Figure 5C). Neutralizing the effects of IL-10 in the primary cultures with an antibody against the IL-10 receptor appeared to have little or no effect on the weak $T$ cell proliferation induced by DCs pulsed with OVA plus zymosan. However, the addition of recombinant IL- 6 induced high levels of proliferation similar to those induced by LPS. Interestingly, the addition of recombinant IL- 6 in conjunction with anti-IL-10 receptor antibody appeared to act in a synergistic manner to enhance $T$ cell proliferation. Thus, zymosan-pulsed DCs induce T cells that appear to be unresponsive to rechallenge with antigen in vitro.

Zymosan induces TGF- $\beta$-producing macrophages but few proinflammatory cytokines in vivo. We wished to determine whether zymosan could also induce $T$ cell unresponsiveness in vivo. First, we determined whether i.v. or i.p. injections of zymosan or LPS resulted in upregulation of the activation markers CD80, CD86, CD40, and class II MHC. Consistent with our previous data (14), CD8 $\alpha^{+}$ and $C D 8 \alpha^{-}$DCs in the spleens expressed greatly enhanced levels of CD80, CD86, CD40, and I-A ${ }^{\mathrm{b}}$ within 4 hours of LPS injection, and these levels remained high up to 10 hours (Figure 6A). In contrast, zymosan was much less effective at inducing these molecules on either DC subset (Figure 6A). We also determined the kind of cytokines induced in the serum by these stimuli. LPS induced abundant levels of IL-12(p40), IL-12(p70), IL-10, IL-6, and TNF- $\alpha$
(Figure 6B). In contrast, zymosan induced little or no IL-12(p40), IL-12(p70), IL-6, or TNF- $\alpha$ (Figure 6B), consistent with its effects on DCs in vitro (Figure 1). However, it did induce significant levels of IL-10, albeit at lower levels than LPS, as well as robust levels of TGF- $\beta$, another potent antiinflammatory cytokine (27) (Figure $7 \mathrm{~A})$. It should be noted that the sera of untreated mice contain significant levels of TGF- $\beta$ (27), but there was a very pronounced and significant induction of TGF- $\beta$ by zymosan above this baseline level. The TGF- $\beta$ present in the serum was biologically active as demonstrated by the phosphorylation of Smad-2 (Figure 7B). The question of exactly what cell types were induced to produce TGF- $\beta$ by zymosan was addressed by 2 different approaches. First, spleen cells were isolated at 1,4 , or 10 hours from mice injected with zymosan and costained with TGF- $\beta$-TRITC and number of other markers, including CD11b-FITC or F4-80-FITC. As indicated in Figure $7 \mathrm{C}$, the majority of TGF- $\beta$-TRITC also stained positively for F4-80-FITC, suggesting that such cells were macrophages. Secondly, we performed confocal analysis of splenic sections from mice that had been injected with zymosan. These experiments also revealed that the principle producers of TGF- $\beta$ were not DCs but rather a subset of $\mathrm{F} 4-80^{+} \mathrm{CD} 11 \mathrm{~b}^{+}$macrophages in the marginal zones and the red pulp of the spleens (Figure 7D).

Injection of zymosan plus antigen into mice results in antigen-specific $T$ cell tolerance. Given the potent induction of the 2 antiinflammatory cytokines IL-10 and TGF- $\beta$ and the weak induction of proinflammatory cytokines in vivo, we determined the type of adaptive immunity induced by zymosan in vivo. We addressed this question using OVA-specific, MHC class II-restricted (I-A $\left.{ }^{\mathrm{b}}\right), \alpha \beta$ TCR transgenic mice (OT-II mice) (14). In these mice, the CD4+ OVA-specific 

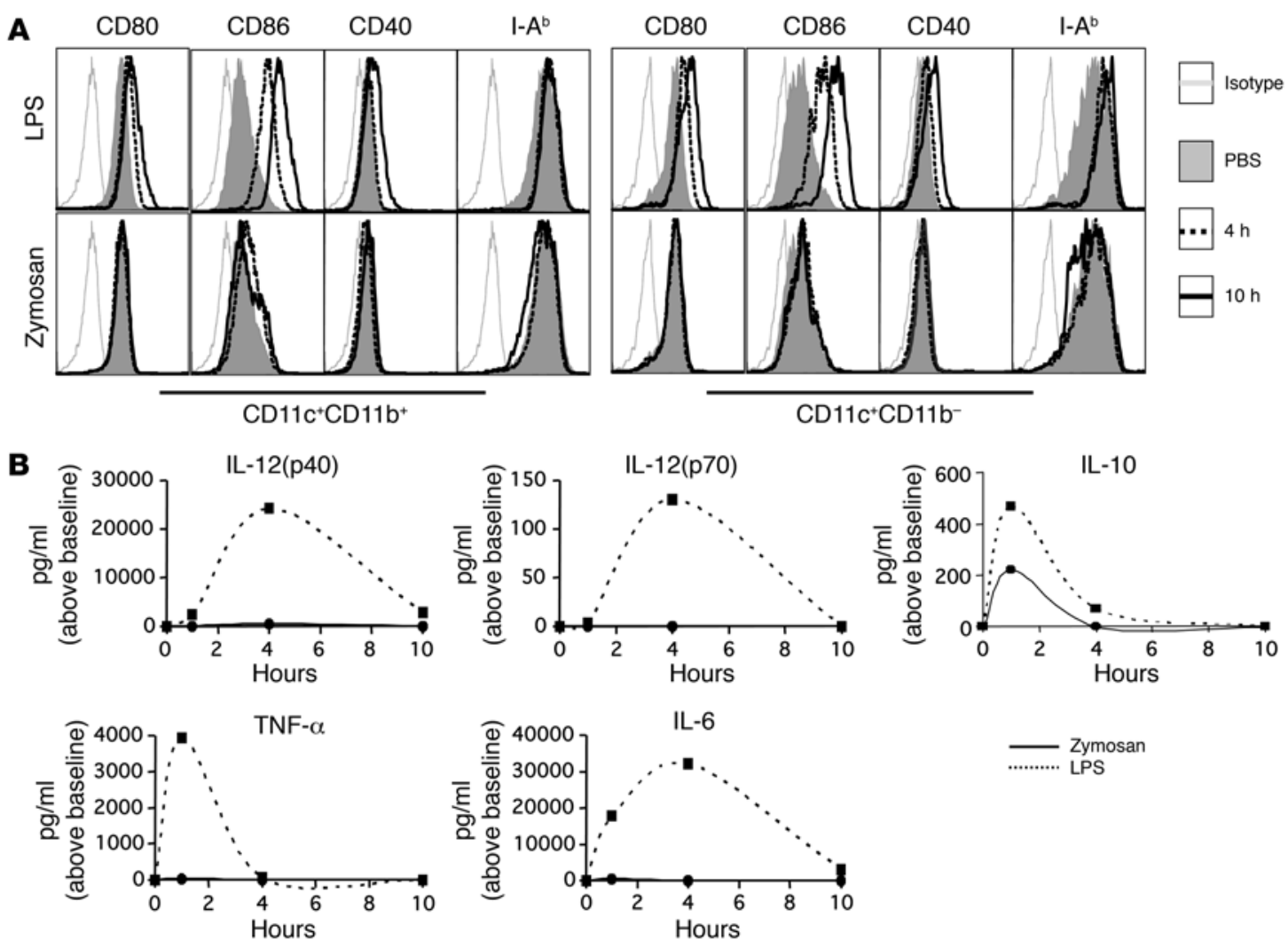

Figure 6

Zymosan is not a potent inducer of costimulatory molecules on splenic DCs or proinflammatory cytokines in vivo. To investigate the effect of zymosan in vivo, C57BL/6 mice were injected with PBS containing either $10 \mu \mathrm{g}$ E. coli LPS or $25 \mu \mathrm{g}$ or $100 \mu \mathrm{g}$ zymosan. (A) Either 4 or 10 hours later, spleens were removed and a small portion digested with collagenase type $4(1 \mathrm{mg} / \mathrm{ml}$; Worthington Biochemical Corp.) in complete DMEM plus $2 \% \mathrm{FBS}$ for 30 minutes at $37^{\circ} \mathrm{C}$. The red blood cells were lysed and the cell suspension washed twice prior to analysis of cell surface-expressed activation markers by flow cytometry. (B) C57BL/6 mice were injected with PBS containing either $10 \mu \mathrm{g}$ E. coli LPS or $25 \mu \mathrm{g}$ zymosan. Blood samples were removed at 1, 4, and 10 hours and serum cytokines analyzed by ELISA.

T cells express V $\alpha 2$ and V $\beta 5$ and, as detailed above, recognize the amino acid 323-339 peptide fragment from OVA. TCR transgenic $T$ cells were adoptively transferred into Thy- 1 congenic B6.PL.Thy- $1^{\text {a }}$ (B6.PL) mice such that they constituted a small but detectable proportion of all $\mathrm{T}$ cells (14). In this system, the fate of OVA-specific, transgenic $T$ cells was followed using the Thy-1.2 antibody, which stains the transferred cells but not the host cells. Cells with the phenotype Thy $-1.2^{+} \mathrm{CD} 4^{+} \mathrm{V} \alpha 2^{+} \mathrm{V} \beta 5^{+}$are considered OVA-specific $\mathrm{CD}^{+} \mathrm{T}$ cells. In some of the experiments, we simply used Thy-1.2 in combination with CD4 to detect the OVA-specific T cells.

The reconstituted mice were injected with $50 \mu \mathrm{g}$ of $\mathrm{OVA}_{323-339}$ peptide alone, $\mathrm{OVA}_{323-339}$ plus LPS, or $\mathrm{OVA}_{323-339}$ plus zymosan i.v. Injection of $\mathrm{OVA}_{323-339}$ alone did not induce any significant clonal expansion of the CD4+ Thy- $1.2^{+}$cells in the spleens of mice although these levels were consistently above those detected in reconstituted mice that had been left untreated, indicating that a limited amount of proliferation of the transferred $T$ cells has taken place in response to OVA in the absence of any adjuvant (Figure 8, $A$ and B). However, LPS significantly enhanced the clonal expansion of CD4 ${ }^{+}$Thy $-1.2^{+}$cells; in contrast, zymosan induced a very weak response although this was still above the response to OVA peptide alone (Figure 8, A and B). There appeared to be no differences in the frequencies of CD $4^{+} \mathrm{Thy}-1.2^{+} \mathrm{CD} 25^{+}$versus $\mathrm{CD} 25^{-} \mathrm{T}$ cells between the various groups (data not shown).
Previous work has shown that productive T cell immunity is elicited only when the antigen is injected with an adjuvant and that injections of soluble antigens only result in a transient and abortive clonal expansion in which antigen-specific $\mathrm{T}$ cells cannot be efficiently restimulated in vitro with protein or peptide $(14,28)$. We thus examined the in vitro proliferative capacity of OVA-specific $\mathrm{T}$ cells from the various cohorts of mice by culturing single-cell suspensions of the spleen with varying concentrations of OVA. As shown in Figure 8C, mice that received OVA $_{323-339}$ plus LPS had greatly enhanced responses compared with those that received $\mathrm{OVA}_{323-339}$ peptide alone; in contrast, mice injected with zymosan had responses that were scarcely different from those observed in mice injected with OVA alone (Figure 8C). Importantly, these differences were not due to the lower frequency of OVA-specific T cells present in the cultures, because as demonstrated by CFSE labeling, $\mathrm{T}$ cells from mice immunized with OVA plus zymosan underwent fewer cell divisions than $T$ cells from mice immunized with OVA plus LPS after in vitro restimulation. (Figure 8D).

Cytokine production by antigen-specific $T$ cells was measured by assaying the culture supernatants from the cultures described above for IFN- $\gamma$, IL-4, IL-5, and IL-10. There were significant differences between mice injected with $\mathrm{OVA}_{323-339}$ peptide alone, $\mathrm{OVA}_{323-339}$ plus LPS, or $\mathrm{OVA}_{323-339}$ plus zymosan (Figure 8E). In cultures from mice injected with $\mathrm{OVA}_{323-339}$ peptide alone, there was little, if any, 

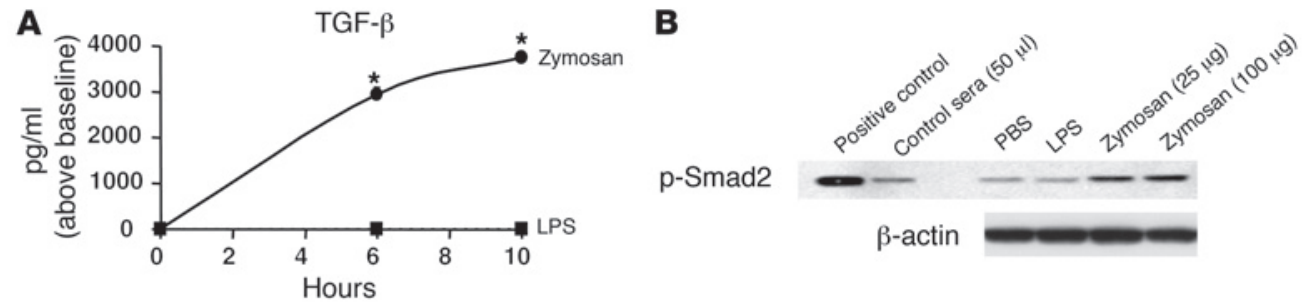

C

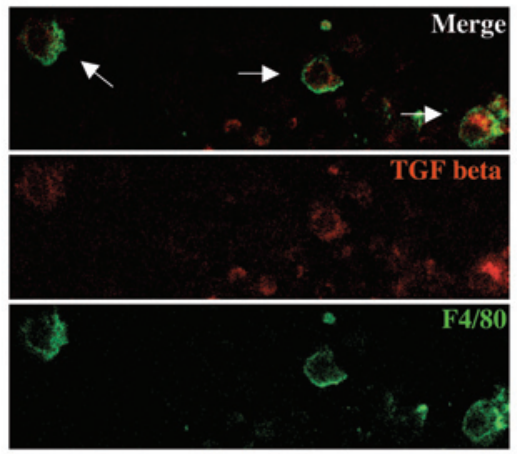

D

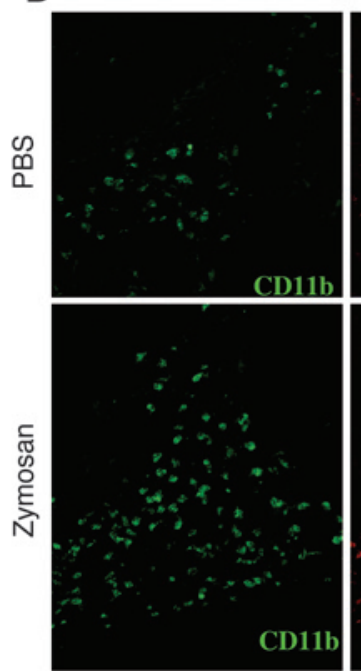

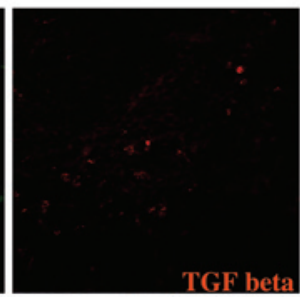
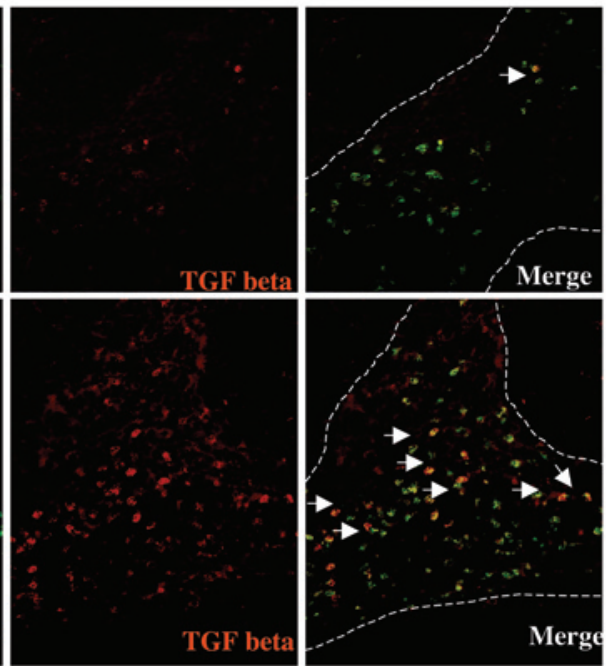

Figure 7

Zymosan induces TGF- $\beta$ from spleen macrophages. (A) C57BL/6 mice were injected i.v. with E. coli LPS (25 $\mu \mathrm{g})$ or zymosan $(25 \mu \mathrm{g})$ and bled at various time points; TGF- $\beta$ induction in the serum was assayed by ELISA. ${ }^{*} P<0.05$, zymosan- versus LPS-treated mice. (B) Biological activity of serum TGF- $\beta$ was determined by stimulating total spleen cells with aliquots of serum and levels of phosphorylation of Smad-2 determined by Western blot. The addition of recombinant TGF- $\beta$ was used as a positive control. (C) Spleen cells were isolated from the mice used in $\mathbf{A}$ at 6 or 10 hours and costained with TGF- $\beta-$ TRITC and F4-80-FITC, then viewed by confocal microscopy. (D) Immunohistological analysis of TGF- $\beta$ production. Splenic sections were prepared from spleens isolated at 1, 4, and 10 hours, stained with CD11b-FITC and TGF- $\beta-$ TRITC, and analyzed by confocal microscopy. Arrows indicate double-positive cells. Original magnification, $\times 40$.

IFN- $\gamma$, IL-4, IL-5, or IL-10. In contrast and consistent with previous reports $(14,28)$, in cultures from mice injected with OVA $_{323-339}$ plus LPS, there were high levels of IFN- $\gamma$ and low levels of IL-4 and IL-5. In striking contrast to this response, in cultures from mice injected with OVA $323-339$ plus zymosan, there were much lower levels of IFN- $\gamma$, IL-4, and IL-5 but high levels of IL-10 (Figure 8E). The fact that IL-10 is only produced in culture when OVA is present suggests that IL-10 induction is dependent on T cells. Therefore, unlike LPS, zymosan induces an antigen-specific $T$ cell response characterized by very weak clonal expansion and in vitro proliferation as well as minimal production of Th 1 and Th 2 cytokines but high levels of IL-10. These characteristics appeared to be indicative of regulatory or tolerogenic responses. We tested this hypothesis further by determining whether injections of $\mathrm{OVA}_{323-339}$ plus zymosan would confer unresponsiveness to a subsequent challenge with $\mathrm{OVA}_{323-339}$ plus a strong adjuvant, such as incomplete Freund's adjuvant (IFA). Mice were primed as before with $\mathrm{OVA}_{323-339}$ OVA $_{323-339}$ plus LPS, or OVA $323-339$ plus zymosan at day 0 and then rechallenged with $\mathrm{OVA}_{323-339}$ plus IFA at day 7. The subsequent clonal expansion of CD4+Thy $1.2^{+}$cells and cytokine production was evaluated. First, it should be noted, the overall magnitude of clonal expansion and cytokine production observed in the secondary responses were markedly lower than those seen in the primary response (Figure $8 \mathrm{~F}$ ), which probably reflects the half-life of the transferred OT-II cells in the recipient. Despite this weaker response, there were striking differences between mice that received OVA $323-339$ plus LPS and OVA $_{323-339}$ plus zymosan. Mice that previously received $\mathrm{OVA}_{323-339}$ plus LPS or no stimulus were competent at responding to $\mathrm{OVA}_{323-339}$ plus IFA. In contrast, mice that previously received $\mathrm{OVA}_{323-339}$ plus zymosan or $\mathrm{OVA}_{323-339}$ were unresponsive to $\mathrm{OVA}_{323-339}$ plus IFA, as evidenced by the negligible clonal expansion and IFN- $\gamma$ and IL-2 secreted in culture (Figure $8 \mathrm{G})$. Interestingly, there was some IL-10 production by the $\mathrm{T}$ cells in the zymosan group (Figure 8G). IL-4, IL-5, and IL-13 could not be detected in any of these cultures (data not shown). Therefore, these data provide evidence that injections of antigen plus zymosan into mice result in a T-helper response that is characterized by very weak clonal expansion and in vitro restimulation and negligible levels of Th1 or Th2 cytokines but high levels of IL-10. Furthermore, such T cells appear to be unresponsive to a subsequent rechallenge with the same antigen plus a gold-standard adjuvant, such as IFA. Further evidence for zymosan inducing unresponsive $T$ cells was observed when LPS and zymosan were coinjected with $\mathrm{OVA}_{323-339}$. As Figure $8 \mathrm{H}$ shows, coinjection of LPS and zymosan resulted in T cells that underwent a reduced number of cell divisions compared with LPSstimulated T cells (Figure $8 \mathrm{H}$ ) and had reduced proliferation (Figure 8I). Taken together, these data suggest that an injection of antigen plus zymosan induces a state of immunological unresponsiveness in antigen-specific T cells. 


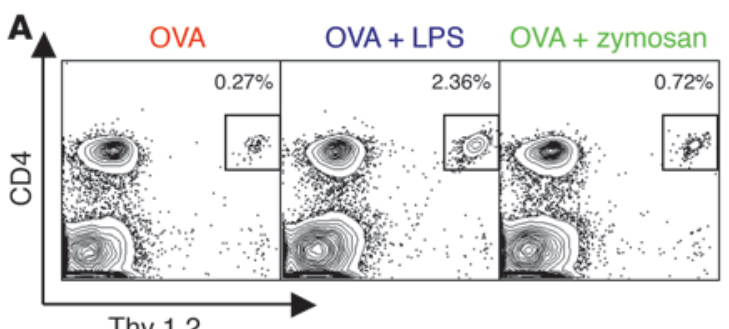

Thy 1.2

D

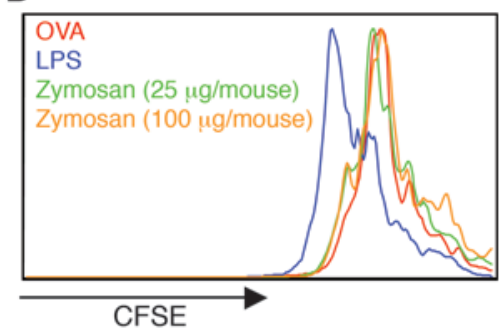

E

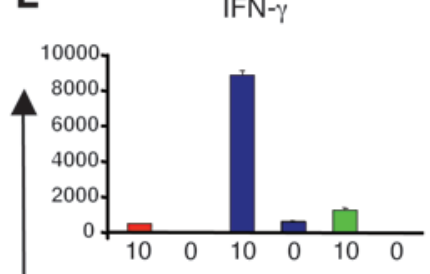

$\mathrm{IL}-4$

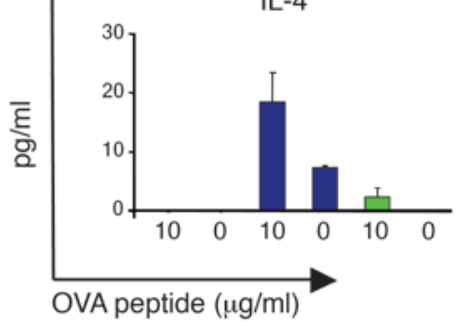

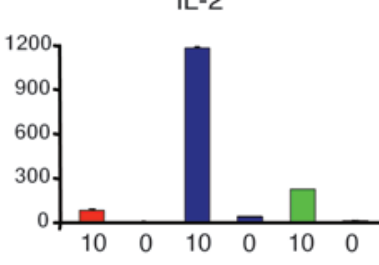

B

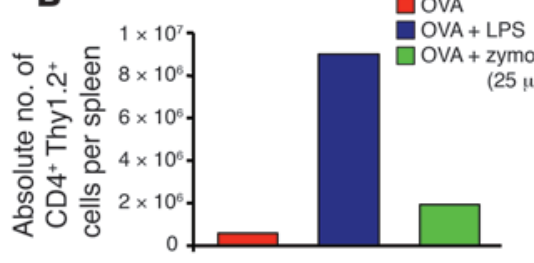

IL-5

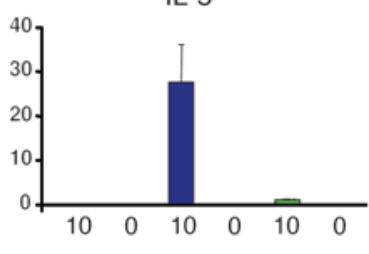

F
C

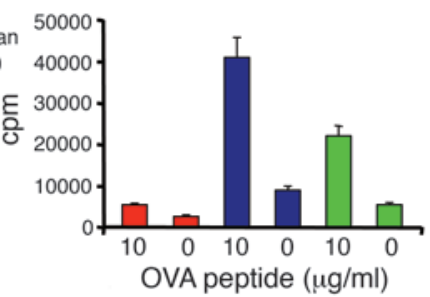

IL-10

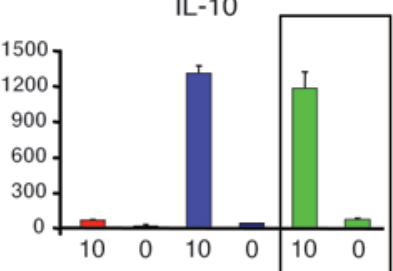

IL-13

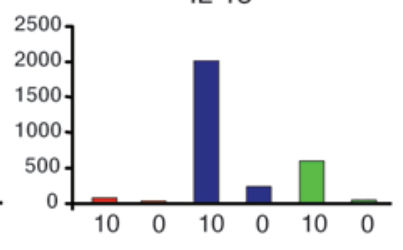

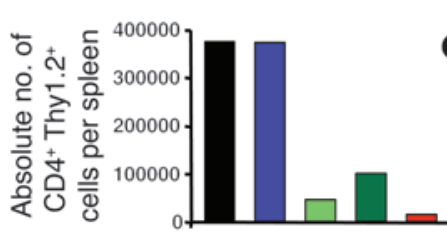

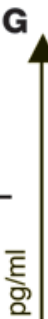
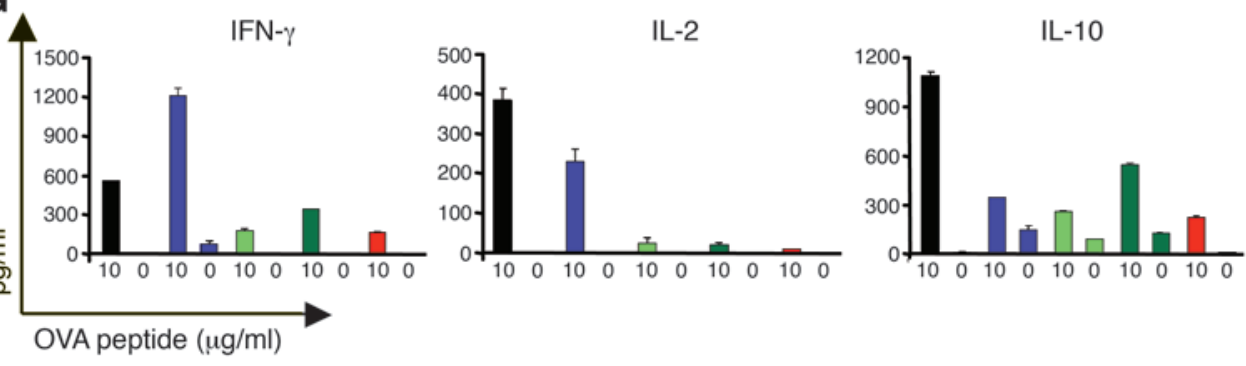

No stimulus, OVAIFA rechallenge OVA + LPS, OVA/IFA rechallenge

H

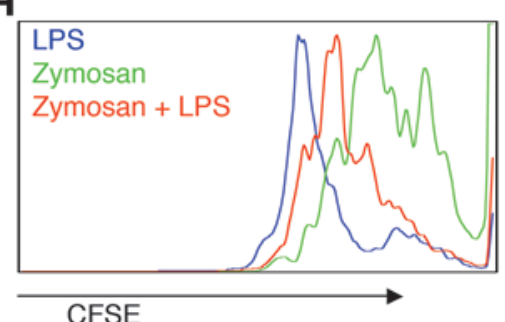

OVA, OVA/IFA rechallenge

OVA + zymosan $(25 \mu \mathrm{g})$, OVA/IFA rechallenge OVA + zymosan $(100 \mu \mathrm{g})$, OVA/IFA rechallenge

I

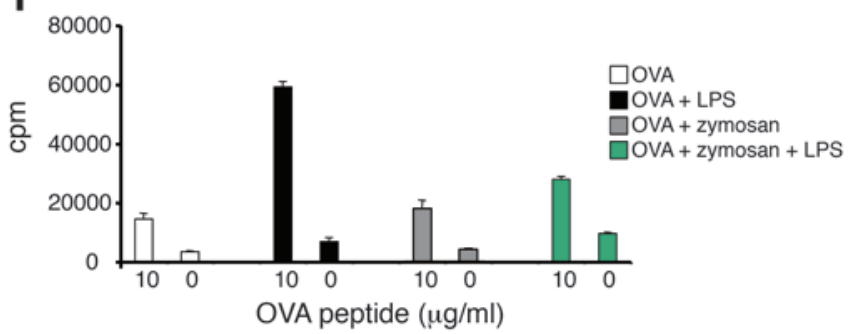

Figure 8

Zymosan induces antigen-specific T cell tolerance in vivo and suppresses the OVA-specific response when coinjected with OVA plus LPS. (A) B6.PL mice reconstituted with OT-II TCR transgenic T cells were injected i.p. with class II-restricted OVA peptide, OVA $323-339$ (50 $\mu \mathrm{g})$ plus LPS (25 $\mu \mathrm{g}), \mathrm{OVA}_{323-339}(50 \mu \mathrm{g})$ plus zymosan (various doses), or OVA $\mathrm{O}_{323-339}$ alone $(50 \mu \mathrm{g})$. Four days later, spleens were removed and clonal expansion of $\mathrm{OVA}_{323-339-\text { specific T cells }(\mathbf{A}) \text { and numbers of OVA }}$ - $23-329-$-specific CD4 $^{+} \mathrm{T}$ cells per spleen (B) were determined by flow cytometry. (C-E) Spleens were isolated 4 days after immunization, and unfractionated spleen cells labeled with CFSE and restimulated in vitro with OVA $\mathrm{A}_{323-339}$ for 72 hours. Proliferation as measured by thymidine uptake (C) or CFSE labeling (D) and cytokine production by ELISA (E) were determined. Zymosan induced T cells that produced only high levels of IL-10 (box). Data are representative of 5 independent experiments. (F and $\mathbf{G})$ Mice immunized as above with $\mathrm{OVA}_{323-339}$ plus LPS or $\mathrm{OVA}_{323-339}$ plus zymosan were rechallenged 10 days later with $\mathrm{OVA}_{323-339}$ in IFA. Four days after challenge, clonal expansion and cytokine production upon in vitro restimulation were evaluated. Data are representative of 4 independent experiments. (H and I) B6.PL mice reconstituted with OT-II TCR transgenic T cells were injected with OVA $\mathrm{O}_{323-339}(50 \mu \mathrm{g})$ plus LPS $(25 \mu \mathrm{g})$, OVA san $(100 \mu \mathrm{g})$, OVA $_{323-339}(50 \mu \mathrm{g})$ plus LPS $(25 \mu \mathrm{g})$ plus zymosan $(100 \mu \mathrm{g})$, or OVA $323-339$ alone $(50 \mu \mathrm{g})$. Four days later, spleens were removed and unfractionated splenocytes were restimulated with OVA $\mathrm{O}_{323-339}$ and (H) CFSE and (I) proliferation of OVA $\mathrm{A}_{323-339-s p e c i f i c} \mathrm{~T}$ cells determined. 
A

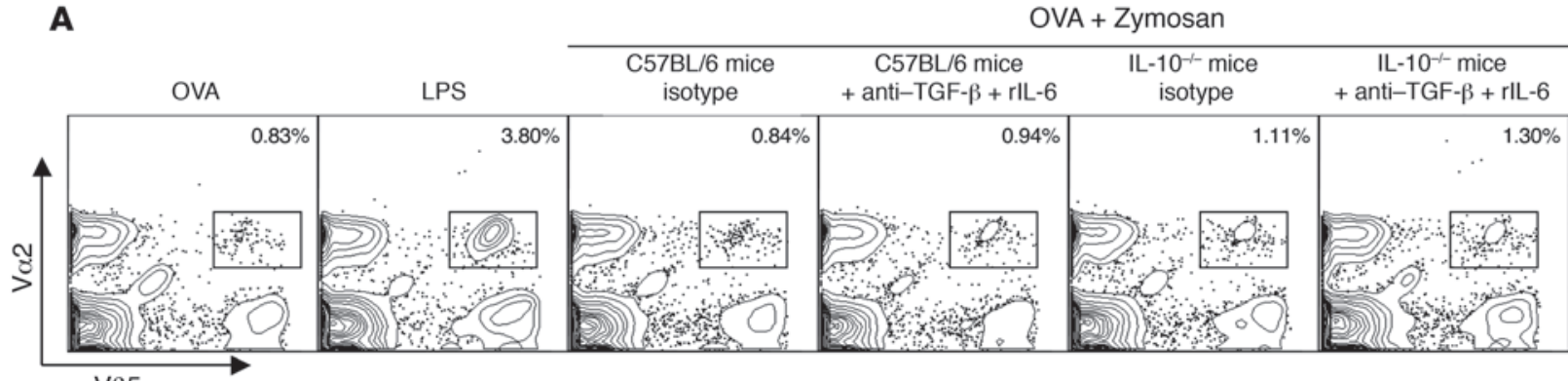

V 85

B C57BL/6 mice

C57BL/6 mice

OVA + zymosan + isotype

OVA + zymosan + anti-TGF- $\beta$ + rlL-6
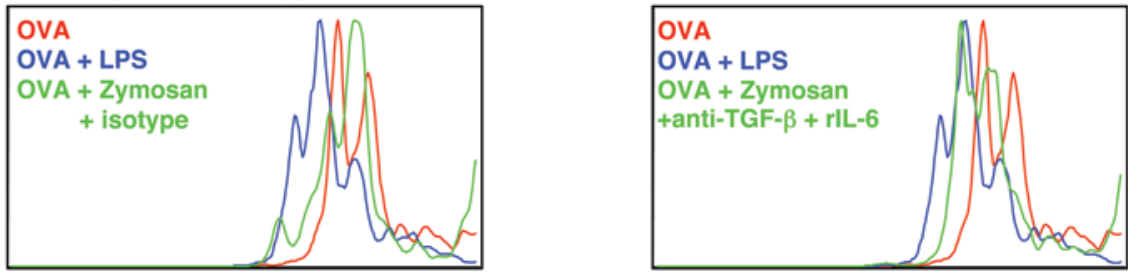

$\mathrm{IL}-10^{-/-}$mice

OVA + zymosan + isotype

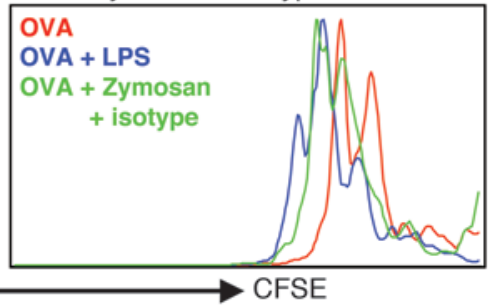

$\mathrm{IL}-10^{-/-}$mice

OVA + zymosan + anti-TGF- $\beta$ + rlL- 6

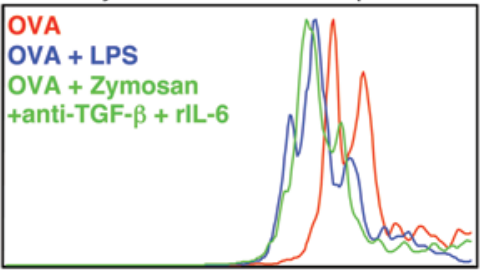

\section{Figure 9}

Impairment of OVA-specific T cell response induced by OVA plus zymosan is dependent on IL-10, TGF- $\beta$, and lack of IL-6. C57BL/6 or IL-10-1- mice were reconstituted with OT-II TCR transgenic T cells on day -1, and the following day, injected with class II-restricted OVA peptide, OVA ${ }_{323-339}$ (50 $\mu \mathrm{g})$ plus LPS $(25 \mu \mathrm{g}), \mathrm{OVA}_{323-339}(50 \mu \mathrm{g})$ plus zymosan $(100 \mu \mathrm{g})$, or OVA $\mathrm{OV}_{323-339}$ alone $(50 \mu \mathrm{g})$. On days -1 , 0 , and 2, appropriate groups of mice were injected with anti-TGF- $\beta$ antibody and recombinant murine IL-6. (A) On day 4, clonal expansion was determined by flow cytometry, and (B) splenocytes were isolated, labeled with CFSE, and restimulated in vitro with $\mathrm{OVA}_{323-339}$ to evaluate proliferation of OVA-specific T cells.

Induction of immunological tolerance by zymosan is dependent on IL-10, TGF- $\beta$, and lack of IL-6. The potential importance of IL-10, TGF- $\beta$, and IL-6 in this induction of immunological unresponsiveness was evaluated by in vivo studies using IL-10-knockout mice, in which TGF- $\beta$ was neutralized with an anti-TGF- $\beta$ antibody, IL- 6 was supplement$\mathrm{ed}$, and the effect on OVA-specific immune responses induced by zymosan plus OVA was assessed (Figure 9). Blocking of TGF- $\beta$, addition of IL-6, and the absence of IL-10 in mice challenged with zymosan plus $\mathrm{OVA}_{323-339}$ each showed small increases in clonal expansion of $\mathrm{OVA}_{323-339} \mathrm{~T}$ cells (Figure 9A) and more impressive increases in the number of cell divisions by those $T$ cells after in vitro restimulation (Figure 9B). The greatest increase, however, was observed when antiTGF- $\beta$ antibody and recombinant IL- 6 were injected into IL-10-/mice challenged with zymosan plus $\mathrm{OVA}_{323-339}$. This injection regime resulted in the largest increase in clonally expanded OVA $323-339-$ specific $\mathrm{T}$ cells compared with the zymosan plus $\mathrm{OVA}_{323-339-\text { challenged }}$ C57BL/6 mice injected with isotype antibody (Figure 9A). Importantly, the combination of all 3 allowed T cells to undergo the same number of divisions that $\mathrm{T}$ cells from LPS plus $\mathrm{OVA}_{323-339}$ underwent upon restimulation in vitro (Figure 9B). Thus, the immunological unresponsiveness induced by zymosan in vivo can be abrogated by blocking TGF- $\beta$ and IL-10 and by the addition of IL- 6 .

\section{Discussion}

In summary, these data suggest that the yeast cell wall, zymosan, previously considered to be a proinflammatory stimulus $(20,27)$, is a potent inducer of IL-10 and TGF- $\beta$ as well as T cell unresponsiveness via multiple mechanisms. First, it induces regulatory-type DCs that produce few proinflammatory cytokines but abundant IL-10 and induce tolerogenic $T$ cell responses. With regard to the mechanism of induction of IL-10, our data suggest a critical role for ERK activation after signaling through both TLR2 and dectin- 1 via a mechanism that is independent of c-Fos. Second, zymosan induces abundant levels of biologically active TGF- $\beta$, another antiinflammatory cytokine, from splenic $\mathrm{F} 4-80^{+}$macrophages in the red pulp. Thus, the dual antiinflammatory effects of IL-10 and TGF- $\beta$ in concert with diminished proinflammatory cytokines likely results in the induction of impaired $\mathrm{T}$ cell responses and tolerance.

These antiinflammatory effects appear to be in contrast to numerous previous reports of the ability of zymosan to induce proinflammatory cytokines such as TNF- $\alpha$ from macrophage cell lines in vitro $(20,27,29,30)$ and to augment inflammatory responses in vivo (30-32). However, in contrast to these studies and in agreement with our study is recent work that suggests that zymosan is also a potent inducer of IL-10 from DCs $(22,33)$. The 


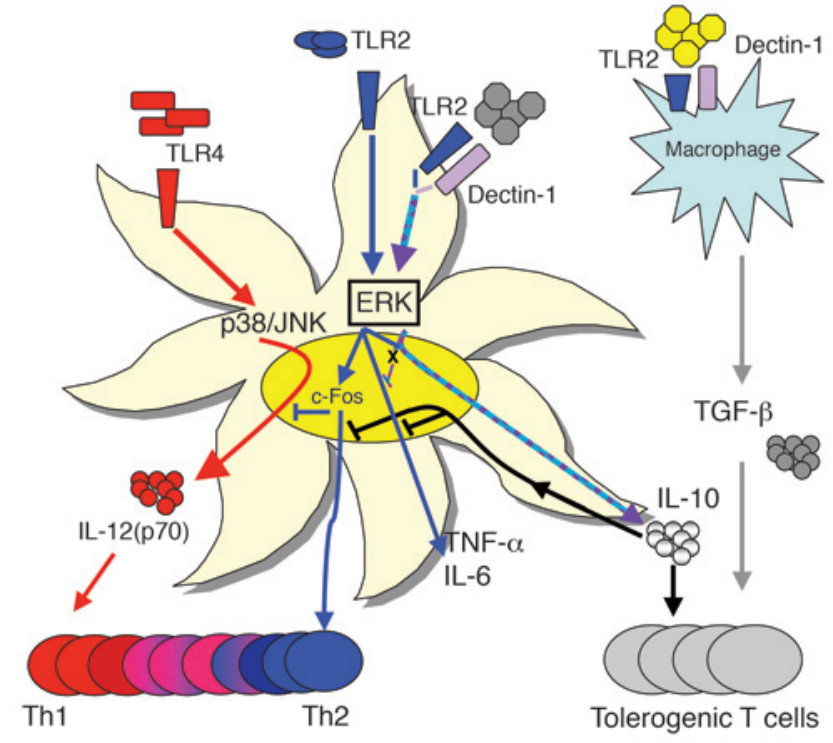

Figure 10

A model for induction of regulatory DCs, macrophages, and immune tolerance by yeast zymosan. Our previous work suggests that Pam-3cys, which signals DCs through TLR2, induces robust IL-10 and IL-6 but little IL-12(p70) $(13,14)$. Induction of IL-10 and impaired IL-12(p70) production appear to be critically regulated by ERK activation, which results in the phosphorylation and stabilization of c-Fos, a repressor of IL-12 $(13,14)$. Such a DC cytokine profile appears to bias toward the Th2 pathway. In the present paper, we demonstrate that zymosan signals to DCs via both TLR2 and dectin-1, inducing the expression of abundant IL-10 but little IL-12(p70) or IL-6. Unlike the case with Pam-3-cys, impairment of IL-12(p70) and induction of c-Fos are not dependent on c-Fos. Induction of IL-10 represses the induction of proinflammatory cytokines, including IL-12, IL-6, and TNF- $\alpha$. In addition to these effects on DCs, zymosan also induces biologically active TGF- $\beta$ in macrophages. In this case, the robust induction of IL-10 and TGF- $\beta$ coupled with impaired induction of IL-6 and IL-12(p70) appears to induce immunological tolerance.

reason for the discrepancies between these studies is not clear but may be due to differences in the subsets of cells being examined (macrophage cell lines in vitro versus bone marrow-derived DCs versus splenic DCs), the dose of zymosan being injected in vivo (a single injection of $10-100 \mu \mathrm{g}$ in our study versus repeated injections of several milligrams in other studies; ref. 32), or the strains of mice used (C57BL/6 in our studies versus Zap-70 knockouts in the study by Yoshitomi et al.; ref. 32). Consistent with a dosedependent effect, we observed that concentrations of zymosan higher than $200 \mu \mathrm{g} / \mathrm{ml}$ (up to $500 \mu \mathrm{g} / \mathrm{ml}$ ) stimulated higher levels of IL-6 and IL-12(p70) from DCs in vitro although even at the highest dose of $500 \mu \mathrm{g} / \mathrm{ml}$, the levels of these cytokines induced were not as much as those induced by LPS (Supplemental Figure 3). Furthermore, the induction of IL-10 appeared to be largely unaffected by these higher concentrations of zymosan. This suggests that the dose of zymosan may in part explain the apparently contradictory observations that zymosan induced proinflammatory versus antiinflammatory cytokines. The mechanism by which such dose differences result in distinct cytokine profiles is of considerable interest. One possibility is that varying the concentration of ligands within zymosan might result in quantitative differences in the affinity, avidity, or threshold of signaling via dectin-1, TLR2, or some other receptor and that this translates into qualitative changes in the signaling pathways and biological response.

The question of which signaling pathway and transcription factors in DCs mediate IL-10 production is of great interest (Figure 10). Our recent work suggests that stimulation of human (13) or murine (14) DCs with the synthetic TLR2 ligand Pam-3-cys results in robust production of IL-10 and IL- 6 but little IL-12(p70). The induction of IL-10 and impaired production of IL-12(p70) appear to be regulated by phosphorylation of ERK MAPK since blocking ERK signaling enhances IL-12(p70) while reducing IL-10 induction $(13,14)$. Furthermore, enhanced ERK induction appears to phosphorylate c-Fos, an AP-1 transcription factor, which represses IL-12 production in DCs $(13,14)$. However, zymosan, which signals through both TLR2 and dectin-1, appears to utilize an overlapping but distinct signaling pathway to mediate its distinctive effects. First, although zymosan is similar to Pam-3-cys in that it induces robust IL-10 and weak IL-12(p70), it differs in its failure to induce abundant IL- 6 . The mechanism underlying this difference is unclear, but one possibility is that IL- 6 production by zymosan is regulated by some TLR2-independent signaling, perhaps via dectin-1. Consistent with this, our data suggest that laminarin results in an enhancement of IL-6 induction by zymosan-treated DCs, particularly at the lower doses of zymosan (Supplemental Figure 4). In contrast, DCs from TLR2-knockout mice produce reduced levels of IL-6. Thus, signaling via TLR2 and dectin-1 appear to exert opposing effects on IL-6 induction by zymosan at the lower doses. Second, although zymosan also induces robust ERK activation, c-Fos does not appear to be involved in regulating IL-12(p70) or IL-10 in zymosan-treated DCs since DCs from c-Fos-knockout mice do not show profound differences in these cytokines (Figure 3). Exactly what transcription factor(s) downstream of ERK might mediate IL-10 induction by zymosan is presently being investigated. Furthermore, which transcription factors might regulate the weak IL-6 and IL-12(p70) responses in zymosan-treated DCs is also being investigated. Whatever such factors might be, it is clear that IL-10 exerts a potent feedback regulatory mechanism that inhibits the production of IL-12, IL- 6 , and TNF- $\alpha$, consistent with other studies $(25,26)$. Interestingly, 2 recent reports suggest that the adaptor protein Syk, which is involved in signaling via dectin-1 in macrophages (29) and DCs (33), mediates the induction of IL-10 (33). However, these reports did not describe the signaling events downstream of Syk. Our present data suggest that ERK activation occurs downstream of Syk and is consistent with similar findings in a different system (34).

Finally, it is intriguing to consider these data from an evolutionary perspective. It has been proposed that pathogens such as HIV, cytomegalovirus, hepatitis C virus, and Mycobacterium tuberculosis target another member of the C-type lectin family, DC-SIGN, to impair DC maturation and adaptive immunity $(15,16)$. One common characteristic of these pathogens is that they can cause chronic infection, during which the Th1/Th2/T regulatory balance is a critical determinant of pathogen persistence in the host. Thus, it is possible that zymosan induces IL-10 and TGF- $\beta$ but suppresses IL-12 and IL- 6 to disarm host immunity. However, since zymosan is not considered to be pathogenic in humans, the evolutionary significance of such an immune evasion mechanism may not be obvious. One possibility is that this mechanism may be equally beneficial for the host and the microbe. Thus, the initial, transient suppression of proinflammatory cytokines might permit the microbe to establish infection in the host, unhindered by the 
host's innate immune system - indeed, the observation that fungal pneumonia can occur after TNF-ablation therapy underscores the critical role of proinflammatory cytokines in the control of infection $(35,36)$. Later, during an established infection, IL-10 and TGF- $\beta$ may serve to keep the inflammation in check, thus avoiding immune pathology, which could be detrimental to the host. Thus, the persistent antiinflammatory response induced by the microbe may be beneficial for both the microbe and host. Such a mechanism may well operate in various chronic infections caused by pathogens, such as HIV, HCV, and M. tuberculosis. Whatever the evolutionary significance, our results highlight several molecular targets, such as TLR2, dectin-1, and ERK, in the therapeutic modulation of innate and adaptive immunity in the context of many pathologic states, such as autoimmunity, allergy, transplantation, and infectious diseases.

\section{Methods}

Mice. C57BL/ 6 mice were purchased from Jackson Laboratory. Male B6.PL mice were purchased from Jackson Laboratory or bred at the Rodent Vivarium of the Yerkes National Primate Center of Emory University. IL-10-/- mice (B6.129P2-Il10 $0^{t m 1 C g n} / \mathrm{J}$ ) were purchased from Jackson Laboratory. B6.129/F1/ $\mathrm{Tac}$ (B6.129) mice were purchased from Taconic). ERK1-knockout (ERK1/-) (37) mice on the C57BL/6 background were kindly provided by G. Landreth (Case Western Reserve University, Cleveland, Ohio, USA) and bred at the Yerkes Rodent Vivarium. c-Fos-knockout mice were kindly provided by L. McCauley (University of Michigan, Ann Arbor, Michigan, USA). OT-II TCR transgenic mice (strain 426-6) (38), generated by W. Heath (Walter and Elisa Hall Institute, Melbourne, Victoria, Australia) and F. Carbone (Monash University, Melbourne, Victoria, Australia), were obtained from J. Kapp (Emory University) and bred at the Yerkes Animal Facility. All mice were aged 6-10 weeks. All animal studies were approved by the Institutional Animal Care and Use Committee of Emory University. For adoptive transfer studies, agematched B6.PL recipients were given $2.5 \times 10^{6}$ OT-II transgenic T cells i.v.

Reagents. Zymosan (Sigma-Aldrich) was boiled for 30 minutes, washed extensively, and stored at $-70^{\circ} \mathrm{C}$. Highly purified Escherichia coli LPS (Strain 25922) was a generous gift from T. Van Dyke. Recombinant human Flt3 ligand was expressed in $\mathrm{CHO}$ cells and purified at the Emory Vaccine Center. Recombinant GM-CSF and IL-4 were purchased from PeproTech. The 3T3-CD40 ligand fibroblasts (39) (a kind gift of P. Hwu, National Cancer Institute, Bethesda, Maryland, USA) were grown in complete DMEM plus $10 \%$ FBS plus $2 \mathrm{mg} / \mathrm{ml} \mathrm{G} 418$ sulphate. Cells were harvested using trypsinEDTA and irradiated at $28.53 \mathrm{~Gy}$ (Gammacell 3000 Elan; MDS Nordion) to prevent further replication. Cells were plated at $4 \times 10^{4}$ cells per well in a 48-well plate and incubated overnight at $37^{\circ} \mathrm{C}$ in a humidified atmosphere of $5 \% \mathrm{CO}_{2}$ in air. Both the MAPK inhibitor U0126 (Calbiochem) and the dectin-1 receptor inhibitor laminarin (from L. digitata; Sigma-Aldrich) were added prior to the addition of stimuli.

Injections. B6.PL mice reconstituted with OT-II TCR transgenic T cells were injected i.p. or i.v. with $50 \mu \mathrm{g}$ MHC class II-restricted OVA peptide (ISQVHAAHAEINEAGR; OVA $323-339$ ) in PBS alone or PBS containing either $25 \mu \mathrm{g}$ E.coli LPS or various doses of zymosan. The OVA peptides were from Brian Evavold (Emory University). To investigate the effect of LPS or zymosan on DCs in vivo, C57BL/6 mice were injected with PBS containing either $25 \mu \mathrm{g}$ E. coli LPS or various doses of zymosan. Six hours later, the spleens were removed and a small portion digested with collagenase type $4(1 \mathrm{mg} / \mathrm{ml}$; Worthington Biochemical Corp. $)$ in complete DMEM plus $2 \% \mathrm{FBS}$ for 30 minutes at $37^{\circ} \mathrm{C}$. The red blood cells were lysed and the cell suspension washed twice prior to analysis of cell surface expression of activation markers by flow cytometry. For rechallenge experiments, OT-II-reconstituted mice were injected with $\mathrm{OVA}_{323-339}$ in PBS alone or
PBS containing LPS or zymosan as detailed above. Additionally, 1 group received $\mathrm{PBS}$ without $\mathrm{OVA}_{323-339}$. Seven days later, all groups were injected i.p. with $\mathrm{OVA}_{323-339}$ emulsified 1:1 in IFA.

Flow cytometry. All antibodies used were from BD Biosciences unless otherwise stated. For analysis of activation of DCs after injection of LPS or zymosan in vivo, rbc-lysed, collagenase-digested spleen cells were incubated at $4^{\circ} \mathrm{C}$ with FITC-conjugated CD11c, APC-conjugated CD $11 \mathrm{~b}$, and PE-conjugated CD80, CD86, CD40, or MHC class II. For analysis of OT-II cells, cell suspensions prepared from spleen cells were incubated at $4^{\circ} \mathrm{C}$ with APC-conjugated CD4 and PE-conjugated Thy 1.2. Levels of phosphorylated ERK (phospho-ERK) were determined by using phosphoERK1 and 2 antibody on fixed and permeabilized ( $90 \%$ methanol) murine CD11 $\mathrm{c}^{+}$DCs or human monocyte-derived DCs as detailed below.

Purification of splenic DCs. CD $11 \mathrm{c}^{+} \mathrm{CD} 11 \mathrm{~b}^{+} \mathrm{CD} 8 \alpha^{-}$and $\mathrm{CD} 11 \mathrm{c}^{+} \mathrm{CD} 11 \mathrm{~b}^{-}$ $\mathrm{CD} 8 \alpha^{+} \mathrm{DC}$ subsets were purified from spleen cell suspensions from mice treated for 9 days with Flt 3 ligand. In brief, spleens from Flt 3 ligand-treated mice were dissected, cut into small fragments, and then digested with collagenase type $4(1 \mathrm{mg} / \mathrm{ml})$ in complete DMEM plus 2\% FBS for $30 \mathrm{~min}$ utes at $37^{\circ} \mathrm{C}$. Cells were washed twice and frozen in FBS plus $10 \%$ DMSO (Sigma-Aldrich). Thawed cells were washed twice, and the CD11 $\mathrm{c}^{+} \mathrm{DCs}$ were enriched using the $\mathrm{CD} 11 \mathrm{c}^{+}$microbeads from Miltenyi Biotec. The resulting purity of CD $11 \mathrm{c}^{+} \mathrm{DCs}$ was approximately $95 \%$. The enriched DCs were stained with FITC-conjugated CD11 $\mathrm{c}$ and PE-conjugated $\mathrm{CD} 11 \mathrm{~b}$ and sorted into the $\mathrm{CD} 11 \mathrm{c}^{+} \mathrm{CD} 11 \mathrm{~b}^{+} \mathrm{CD} 8 \alpha^{-}$and $\mathrm{CD} 11 \mathrm{c}^{+} \mathrm{CD} 11 \mathrm{~b}-\mathrm{CD} 8 \alpha^{+} \mathrm{DC}$ subsets using a high-speed modular flow cytometer (MoFlo; Cytomation Inc.).

In vitro cultures of murine DCs and T cells. CD $11 \mathrm{c}^{+}$-enriched DCs or $\mathrm{CD} 11 \mathrm{c}^{+} \mathrm{CD} 11 \mathrm{~b}^{+}$and $\mathrm{CD} 11 \mathrm{c}^{+} \mathrm{CD} 11 \mathrm{~b}^{-} \mathrm{DC}$ subsets were plated with either LPS or zymosan in complete DMEM plus 10\% FBS onto a confluent layer of irradiated CD40 ligand fibroblasts to induce CD40 ligand triggering. MAPK inhibitor U0126 $(10 \mu \mathrm{M})$ or laminarin $(200 \mu \mathrm{g} / \mathrm{ml})$ was added prior to the addition of stimuli to the $\mathrm{CD} 11 \mathrm{c}^{+}$-enriched DC. Cultures were incubated in a humidified atmosphere of $5 \% \mathrm{CO}_{2}$ in air at $37^{\circ} \mathrm{C}$ for $24-48$ hours. The culture supernatants were collected and assayed for the presence of IL-12(p40), IL-12(p70), IL-10, IL-6, and TNF- $\alpha$. For in vitro T cell assays, CD $11 \mathrm{c}^{+}$-enriched DCs were cultured with naive OT-II CD4 ${ }^{+} \mathrm{CD} 62 \mathrm{~L}^{+}$and OVA peptide and either LPS $(1 \mu \mathrm{g} / \mathrm{ml})$ or zymosan $(10 \mu \mathrm{g} / \mathrm{ml})$. Various concentrations of recombinant murine IL-6 (PeproTech) or anti-IL-10 receptor antibody (BD Biosciences) were added to appropriate wells. Either 2 or 5 days later, the T cells were restimulated with plate-bound anti-CD3 $(10 \mu \mathrm{g} / \mathrm{ml})$ and anti-CD28 $(2 \mu \mathrm{g} / \mathrm{ml})$ for an additional 3 days. Cultures were pulsed with $1 \mu \mathrm{Ci}\left[{ }^{3} \mathrm{H}\right]$ thymidine for 12 hours, and incorporation of the radionucleotide was measured by $\beta$-scintillation spectroscopy. Four days after in vivo priming with OVA peptide plus ligand, rbc-depleted spleen cells were cultured in triplicate in 96-well round-bottomed plates $\left(1 \times 10^{6}\right.$ cells/well $)$ in complete DMEM plus 10\% FBS together with different concentrations of OVA peptide. Proliferative responses were assessed after 72 hours of culture in a humidified atmosphere of $5 \% \mathrm{CO}_{2}$ in air at $37^{\circ} \mathrm{C}$. Cultures were pulsed with $1 \mu \mathrm{Ci}\left[{ }^{3} \mathrm{H}\right]$ thymidine for 12 hours, and incorporation of the radionucleotide was measured by $\beta$-scintillation spectroscopy. For cytokine assays, aliquots of culture supernatants were removed after 90 hours, pooled, and assayed for the presence of IL-2, IL-4, IL-5, IL-13, IL-10, and IFN- $\gamma$ by ELISA.

In vivo blocking and addition of recombinant cytokines. OT-II T cells were transferred into $\mathrm{C} 57 \mathrm{BL} / 6$ or IL-10/- mice as described above. On the same day, anti-TGF- $\beta$ antibody (150 $\mu$ g per mouse; Harlan) or $500 \mathrm{ng}$ recombinant murine IL-6 (PeproTech,) was injected i.p. Mice were challenged with $50 \mu \mathrm{g} \mathrm{OVA}_{323-339}$ in PBS alone or PBS containing either $25 \mu \mathrm{g}$ E. coli LPS or $100 \mu \mathrm{g}$ zymosan the next day. Anti-TGF- $\beta$ antibody or recombinant IL- 6 were injected the same day and again 2 days later.

Measurement of cytokine production. IL-2, IL-4, IL-5, IL-6, IL-12(p40), IL-12(p70), IL-10, TNF- $\alpha$, and IFN- $\gamma$ in cell culture supernatants or serum 
were quantitated by ELISA antibody sets from BD Biosciences, and IL-13 was measured by an ELISA kit from R\&D Systems. TGF- $\beta$ was measured by an ELISA kit from BioSource International.

Isolation, culture, and phenotyping of human monocyte-derived DCs. CD14+ monocytes were enriched from peripheral blood mononuclear cells and cultured for 6 days with recombinant human GM-CSF at $100 \mathrm{ng} / \mathrm{ml}$ plus recombinant human IL-4 at $20 \mathrm{ng} / \mathrm{ml}$. At day 6 , the cultures consisted uniformly of $\mathrm{CD} 1 \mathrm{a}^{+} \mathrm{CD} 14-\mathrm{HLA}-\mathrm{DR}^{+} \mathrm{CD} 11 \mathrm{c}^{+}$cells, which were negative for CD83. These immature DCs were pulsed with LPS or zymosan for 24-48 hours. Phenotype was determined by flow cytometry using a FACScalibur (BD Biosciences). Briefly, gated CD $1 \mathrm{a}^{+} \mathrm{CD} 14^{-} \mathrm{CD} 11 \mathrm{c}^{+} \mathrm{HLA}-\mathrm{DR}{ }^{+} \mathrm{DCs}$ were analyzed for the expression of CD80, CD86, or CD83. Cytokine production was measured by ELISA.

Evaluation of ERK MAPK signaling. This was done using commercially available ELISA kits (BioSource International), or flow cytometry. Briefly, on day 6 , immature, human monocyte-derived DCs $\left(2 \times 10^{6}\right)$ were cultured for the indicated times with the stimuli. ELISA assays were performed according to manufacturer instructions. Flow cytometric evaluation of phospho-ERK in DCs was determined by stimulating day-6, human monocyte-derived DCs for 15 minutes, 1 hour, and 4 hours with LPS or zymosan and then fixing the cells in $2 \%$ paraformaldehyde (10\% ultrapure EM Grade; Polysciences Inc.) for 10 minutes at $37^{\circ} \mathrm{C}$. After washing, the cells were permeabilized with freshly prepared $90 \%$ ice-cold methanol for 30 minutes on ice. Then the cells were washed twice in staining buffer ( $3 \%$ FCS in PBS) and labeled with phospho-ERK for 30 minutes on ice, then washed in staining buffer and labeled using FITC-labeled goat-anti-rabbit Ig. Flow cytometry was done on FACSCalibur. Detection of phospho-ERK1 and 2 was detected in murine $\mathrm{CD} 11 \mathrm{c}^{+}$in a similar fashion.

Detection of biological activity of serum TGF- $\beta$. Spleen cells were incubated with serum from mice previously challenged with PBS, LPS $(25 \mu \mathrm{g} / \mathrm{ml})$, or zymosan $(25 \mu \mathrm{g} / \mathrm{ml}$ or $100 \mu \mathrm{g} / \mathrm{ml})$. Lysates of cells treated with $2.5 \mathrm{ng}$ TGF- $\beta 1 / \mathrm{ml}$ in RPMI medium with $0.5 \%$ fetal bovine serum were separated on $8 \%$ tris-glycine gels (Invitrogen Corp.). Immunoblotting was performed with an anti-phosho-Smad2, rabbit polyclonal antibody (Upstate USA Inc.) followed by a 1:10,000 dilution of goat-anti-rabbit secondary (Jackson ImmunoResearch Laboratories Inc.) and visualized with Super Signal (Pierce Biotechnology).

Immunohistological analysis of cells: cytospins. Single-cell suspensions of spleens were prepared as described elsewhere. Approximately $10^{6}$ splenocytes were cultured for 6 hours with brefeldin A (BD Biosciences) per the manufacturer's instructions. After blocking with Fc block (clone 2.4G2; BD Biosciences), surface staining was done using the FITC-labeled anti-mouse F4/80 (1:100) (Clone CI:A3-1; CALTAG Laboratories) followed by permeabilization using the BD Biosciences cytofix/cytoperm kit per the manufac- turer's recommendations. Staining for intracellular TGF- $\beta$ was carried out using a rabbit polyclonal anti-TGF- $\beta$ antibody $(5 \mu \mathrm{g} / \mathrm{ml})$; kindly provided by Kathleen Flanders, National Cancer Institute, Bethesda, Maryland, USA) followed by TRITC-labeled anti-rabbit IgG (Santa Cruz Biotechnology Inc.). Normal rabbit IgG (Santa Cruz Biotechnology Inc.) was used as an isotype control, and no detectable staining was observed. Stained cells were spun onto glass slides using a cytospin (Shandon Cytopsin 2) and mounted with Vectashield Hard set (Vector Laboratories). Three images were acquired for each field using a Zeiss Axiovert LSM-410 confocal microscope (showing FITC, TRITC, and both dyes simultaneously on the cells).

Frozen section immunofluorescence analysis. Mice were sacrificed at the indicated time points, and spleens were snap frozen in tissue-freezing medium (Triangle Biomedical Sciences). Immunofluorescence staining was carried out on $6-\mu \mathrm{m}$ sections. Sections were air dried and fixed with a 1:1 acetone methanol mixture at $-20^{\circ} \mathrm{C}$ for 10 minutes followed by blocking for $30 \mathrm{~min}$ utes (PBS with 0.1\% Tween 20 and 1:200 Fc block). Sections were incubated with a 1:100 dilution of FITC-labeled anti-mouse CD11b (clone M1/70) and rabbit polyclonal TGF- $\beta$ antibody $(5 \mu \mathrm{g} / \mathrm{ml})$ overnight at $4{ }^{\circ} \mathrm{C}$ followed by washing and incubation with TRITC-labeled anti-rabbit IgG (Santa Cruz Biotechnology Inc.) at room temperature for 1 hour (antibody dilutions were performed in PBS containing 1:200 Fc Block). Normal rabbit IgG (Santa Cruz Biotechnology Inc.) was used as an isotype control, and staining was undetectable with this antibody. Washed sections were mounted with Vectashield Hard set (Vector Laboratories). Three images were acquired for each field using a Zeiss Axiovert LSM-410 confocal microscope (showing FITC, TRITC, and both dyes simultaneously on the cells).

Statistics. Statistical significance of differences in means \pm SD of cytokines released by cells from different groups was calculated using the 2-tailed Student's $t$ test. $P$ values less than 0.05 were considered statistically significant.

\section{Acknowledgments}

This work was supported by NIH grants AI0564499, AI048638, AI05726601, DK057665, AI057157, and AI05726601. We thank Mike Hulsey for help with the FACS sorting experiments and Pearl Backman for help with preparation of the manuscript.

Received for publication October 24, 2005, and accepted in revised form January 24, 2006.

Address correspondence to: Bali Pulendran, Emory Vaccine Center, 954 Gatewood Road, Atlanta, Georgia 30329, USA. Phone: (404) 727-8945; Fax: (404) 727-8199; E-mail: bpulend@rmy.emory.edu.

\section{S. Dillon and S. Agrawal contributed equally to this work.}

1. Janeway, C.A., Jr. 1989. Approaching the asymptote? Evolution and revolution in immunology. Cold Spring Harb. Symp. Quant. Biol. 54:1-13.

2. Shortman, K., and Liu, Y.J. 2002. Mouse and human dendritic cell subtypes. Nat. Rev. Immunol. 2:151-161.

3. Pulendran, B. 2004. Modulating vaccine responses with dendritic cells and Toll-like receptors. Immunol. Rev. 199:227-250.

4. Pasare, C., and Medzhitov, R. 2004. Toll-like receptors and acquired immunity. Semin. Immunol. 16:23-26.

5. Takeda, K., Kaisho, T., and Akira, S. 2003. Toll-like receptors. Annu. Rev. Immunol. 21:335-376.

6. van Kooyk, Y., and Geijtenbeek, T.B. 2003. DCSIGN: escape mechanism for pathogens. Nat. Rev. Immunol. 3:697-709.

7. Cambi, A., and Figdor, C.G. 2003. Dual function of C-type lectin-like receptors in the immune system. Curr. Opin. Cell Biol. 15:539-546.
8. Gordon, S. 2002. Pattern recognition receptors: doubling up for the innate immune response. Cell. 111:927-930.

9. Toshchakov, V., et al. 2002. TLR4, but not TLR2, mediates IFN-beta-induced STAT1alpha/betadependent gene expression in macrophages. Nat. Immunol. 3:392-398.

10. Ito, T., et al. 2002. Interferon-alpha and interleukin-12 are induced differentially by Toll-like receptor 7 ligands in human blood dendritic cell subsets. J. Exp. Med. 195:1507-1512.

11. Hoebe, K., et al. 2003. Identification of Lps2 as a key transducer of MyD88-independent TIR signalling. Nature. 424:743-748.

12. Yamamoto, M., et al. 2003. Role of adaptor TRIF in the MyD88-independent toll-like receptor signaling pathway. Science. 301:640-643.

13. Agrawal, S., et al. 2003. Cutting edge: different Tolllike receptor agonists instruct dendritic cells to induce distinct Th responses via differential modu- lation of extracellular signal-regulated kinase-mitogen-activated protein kinase and c-Fos. J. Immunol. 171:4984-4989.

14. Dillon, S., et al. 2004. A Toll-like receptor 2 ligand stimulates Th2 responses in vivo, via induction of extracellular signal-regulated kinase mitogen-activated protein kinase and c-Fos in dendritic cells. J. Immunol. 172:4733-4743.

15. Engering, A., Geijtenbeek, T.B., and van Kooyk, Y. 2002. Immune escape through C-type lectins on dendritic cells. Trends Immunol. 23:480-485.

16. Brown, G.D., and Gordon, S. 2001. Immune recognition. A new receptor for beta-glucans. Nature. 413:36-37.

17. Brown, G.D., et al. 2002. Dectin-1 is a major betaglucan receptor on macrophages. J. Exp. Med. 196:407-412.

18. Reid, D.M., et al. 2004. Expression of the beta-glucan receptor, Dectin-1, on murine leukocytes in situ correlates with its function in pathogen rec- 
ognition and reveals potential roles in leukocyte interactions. J. Leukoc. Biol. 76:86-94.

19. Yokota, K., Takashima, A., Bergstresser, P.R., and Ariizumi, K. 2001. Identification of a human homologue of the dendritic cell-associated C-type lectin-1, dectin-1. Gene. 272:51-60

20. Gantner, B.N., Simmons, R.M., Canavera, S.J., Akira, S., and Underhill, D.M. 2003. Collaborative induction of inflammatory responses by dectin-1 and Toll-like receptor 2. J. Exp. Med. 197:1107-1117.

21. Manickasingham, S.P., Edwards, A.D., Schulz, O. and Reis e Sousa, C. 2003. The ability of murine dendritic cell subsets to direct $T$ helper cell differentiation is dependent on microbial signals. EurJ. Immunol. 33:101-107.

22. Edwards, A.D., et al. 2002. Microbial recognition via Toll-like receptor-dependent and -independent pathways determines the cytokine response of murine dendritic cell subsets to CD40 triggering. J. Immunol. 169:3652-3660.

23. Pasare, C., and Medzhitov, R. 2003. Toll pathway-dependent blockade of CD4+CD25+ T cellmediated suppression by dendritic cells. Science. 299:1033-1036.

24. Underhill, D.M., et al. 1999. The Toll-like receptor 2 is recruited to macrophage phagosomes and discriminates between pathogens. Nature. 401:811-815.

25. Murray, P.J. 2005. The primary mechanism of the
IL-10-regulated antiinflammatory response is to selectively inhibit transcription. Proc. Natl. Acad. Sci. U. S. A. 102:8686-8691.

26. Hawrylowicz, C.M., and O'Garra, A. 2005. Potential role of interleukin-10-secreting regulatory $\mathrm{T}$ cells in allergy and asthma. Nat. Rev. Immunol. 5:271-283.

27. Sanguedolce, M.V., Capo, C., Bongrand, P., and Mege, J.L. 1992. Zymosan-stimulated tumor necrosis factor-alpha production by human monocytes. Down-modulation by phorbol ester. J. Immunol. 148:2229-2236.

28. Kearney, E.R., Pape, K.A., Loh, D.Y., and Jenkins, M.K. 1994. Visualization of peptide-specific T cell immunity and peripheral tolerance induction in vivo. Immunity. 1:327-339.

29. Underhill, D.M., Rossnagle, E., Lowell, C.A., and Simmons, R.M. 2005. Dectin-1 activates Syk tyrosine kinase in a dynamic subset of macrophages for reactive oxygen production. Blood. 106:2543-2550.

30. Brown, G.D., and Gordon, S. 2003. Fungal betaglucans and mammalian immunity. Immunity. 19:311-315.

31. Keystone, E.C., Schorlemmer, H.U., Pope, C., and Allison, A.C. 1977. Zymosan-induced arthritis: a model of chronic proliferative arthritis following activation of the alternative pathway of complement. Arthritis Rheum. 20:1396-1401.

32. Yoshitomi, H., et al. 2005. A role for fungal betaglucans and their receptor Dectin-1 in the induc- tion of autoimmune arthritis in genetically susceptible mice. J. Exp. Med. 201:949-960.

33. Rogers, N.C., et al. 2005. Syk-dependent cytokine induction by Dectin- 1 reveals a novel pattern recognition pathway for $\mathrm{C}$ type lectins. Immunity. 22:507-517.

34. Sedlik, C., et al. 2003. A critical role for Syk protein tyrosine kinase in Fc receptor-mediated antigen presentation and induction of dendritic cell maturation. J. Immunol. 170:846-852.

35. Romani, L. 2004. Immunity to fungal infections. Nat. Rev. Immunol. 4:1-23.

36. Bozza, S., et al. 2004. Dendritic cell-based vaccination against opportunistic fungi. Vaccine. 22:857-864.

37. Selcher, J.C., Nekrasova, T., Paylor, R., Landreth, G.E., and Sweatt, J.D. 2001. Mice lacking the ERK1 isoform of MAP kinase are unimpaired in emotional learning. Learn. Mem. 8:11-19.

38. Barnden, M.J., Allison, J., Heath, W.R., and Carbone, F.R. 1998. Defective TCR expression in transgenic mice constructed using cDNA-based alpha- and beta-chain genes under the control of heterologous regulatory elements. Immunol. Cell Biol. 76:34-40.

39. Lapointe, R., Toso, J.F., Butts, C., Young, H.A., and Hwu, P. 2000. Human dendritic cells require multiple activation signals for the efficient generation of tumor antigen-specific T lymphocytes. Eur J. Immunol. 30:3291-3298. 